\title{
Radiative transfer models for retrieval of cloud parameters from EPIC/DSCOVR measurements
}

\author{
Víctor Molina García*, Sruthy Sasi, Dmitry S. Efremenko, Adrian Doicu, Diego Loyola \\ Deutsches Zentrum für Luft- und Raumfahrt (DLR), Institut für Methodik der Fernerkundung (IMF), 82234 Oberpfaffenhofen, Germany
}

\begin{abstract}
In this paper we analyze the accuracy and efficiency of several radiative transfer models for inferring cloud parameters from radiances measured by the Earth Polychromatic Imaging Camera (EPIC) on board the Deep Space Climate Observatory (DSCOVR). The radiative transfer models are the exact discrete ordinate and matrix operator methods with matrix exponential, and the approximate asymptotic and equivalent Lambertian cloud models. To deal with the computationally expensive radiative transfer calculations, several acceleration techniques such as, for example, the telescoping technique, the method of false discrete ordinate, the correlated $k$-distribution method and the principal component analysis (PCA) are used. We found that, for the EPIC oxygen A-band absorption channel at $764 \mathrm{~nm}$, the exact models using the correlated $k$-distribution in conjunction with PCA yield an accuracy better than $1.5 \%$ and a computation time of $18 \mathrm{~s}$ for radiance calculations at 5 viewing zenith angles.
\end{abstract}

Keywords: backscattering, PCA, correlated $k$-distribution, asymptotic theory, EPIC

\section{Introduction}

The Earth Polychromatic Imaging Camera (EPIC) on board the Deep Space Climate Observatory (DSCOVR) was designed to measure the atmosphere and surface properties over the whole sunlit face of the Earth from the Lagrange point $\mathrm{L}_{1}$ (a gravity-neutral position at $1.5 \times 10^{6} \mathrm{~km}$ away from the Earth). DSCOVR is placed in a Lissajous orbit around the $\mathrm{L}_{1}$ point, and provides a unique angular perspective at almost backward direction with scattering angles approximately between $168^{\circ}$ and $176^{\circ}$ (Fig. 1). EPIC scans the entire sunlit face of the Earth at a $2048 \times 2048$ pixel resolution, with a pixel size of $12 \times 12 \mathrm{~km}^{2}$ at the image center. The instrument has 10 spectral channels ranging from the ultraviolet to the near-infrared. Four of them are located in the oxygen A- and B- bands: two absorption channels centered at $688 \mathrm{~nm}$ and $764 \mathrm{~nm}$ with bandwidths of $0.8 \mathrm{~nm}$ and $1.0 \mathrm{~nm}$, respectively, and two continuum channels centered at $680 \mathrm{~nm}$ and $780 \mathrm{~nm}$ with bandwidths of $3.0 \mathrm{~nm}$ and $2.0 \mathrm{~nm}$ (Table 1). These channels are used for monitoring the vegetation condition [1], the aerosol layer height and optical depth [2], and the cloud height [3].

The radiative transfer for retrieval of cloud parameters involves, in addition to cloud scattering and absorption, gas absorption and molecular Rayleigh scattering. Usually, it is necessary to consider spectral regions containing several overlapping lines with intensities varying over many orders of magnitude. An accurate method for computing the radiative transfer in a molecular atmosphere relies on line-by-line (LBL) calculations. However, LBL calculations are in most cases too computationally expensive to be used directly in online and even sometimes

\footnotetext{
${ }^{*}$ Corresponding author

Email address: Victor.MolinaGarcia@dlr.de (Víctor Molina García)
}

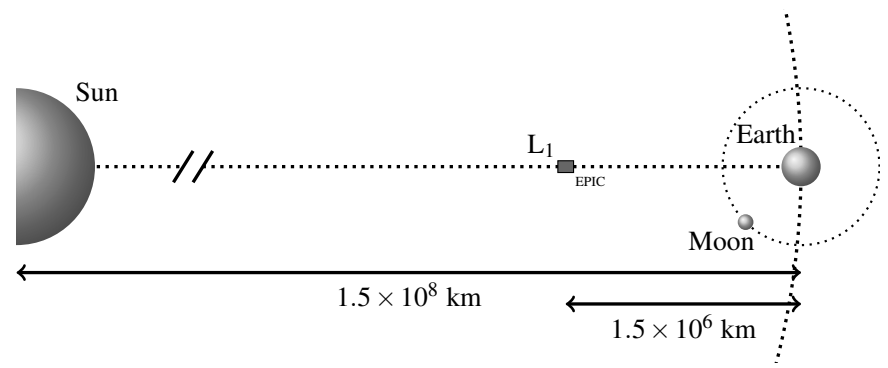

Figure 1: Illustration of EPIC / DSCOVR geometry (distances are not to scale).

in offline retrieval algorithms. This prompts us to use exact or approximate radiative transfer models endowed with acceleration techniques. As approximate models, asymptotic [4-6] and equivalent Lambertian cloud models [7-9] are frequently used. In the category of acceleration techniques we include here the correlated $k$-distribution method [10,11], the radiance sampling method [12], the optimal spectral mapping method [13] and dimensionality reduction techniques. In the latter case, principal component analysis (PCA) is used to map the spectral radiances into a lower-dimensional subspace in which the inversion is performed [14, 15], or to reduce the dimensionality of the optical properties [16-18]. In addition, the telescoping technique $[19,20]$ and the method of false discrete ordinate [21-24] can be used to speed up radiative transfer calculations.

For the EPIC instrument, the retrieval is more challenging due to the singular geometry of the radiative transfer problem. Given a particle with a certain size, the scattering phase function shows considerable structure and resonances. When averaged over a size distribution of an ensemble of particles, these features are almost smoothed out, but they are still present in 
Table 1: Description of EPIC channels, adapted from EPIC's main website (https://epic.gsfc.nasa.gov/epic).

\begin{tabular}{cccc}
\hline Channel & Central wavelength $/ \mathbf{n m}$ & Full width $/ \mathbf{n m}$ & Primary application \\
\hline 1 & $317.5 \pm 0.1$ & $1.0 \pm 0.2$ & Ozone, sulfur dioxide \\
2 & $325.0 \pm 0.1$ & $2.0 \pm 0.2$ & Ozone \\
3 & $340.0 \pm 0.3$ & $3.0 \pm 0.6$ & Ozone, aerosols \\
4 & $388.0 \pm 0.3$ & $3.0 \pm 0.6$ & Aerosols, clouds \\
5 & $443.0 \pm 1.0$ & $3.0 \pm 0.6$ & Aerosols, clouds \\
6 & $551.0 \pm 1.0$ & $3.0 \pm 0.6$ & Aerosols \\
7 & $680.0 \pm 0.2$ & $3.0 \pm 0.6$ & Aerosols, clouds, vegetation \\
8 & $687.75 \pm 0.20$ & $0.80 \pm 0.20$ & Aerosols, clouds, vegetation \\
9 & $764.0 \pm 0.2$ & $1.0 \pm 0.2$ & Cloud height \\
10 & $779.5 \pm 0.3$ & $2.0 \pm 0.4$ & Clouds, vegetation \\
\hline
\end{tabular}

the backward and forward glories, at scattering angles around $180^{\circ}$ and $0^{\circ}$, and in the rainbow region, at around $140^{\circ}$ [25]. For an accurate description of the specific features of the single scattering properties in the backward direction, a large number of discrete ordinates is required, even when the delta-M method [26] is used.

The aim of this paper is to analyze the accuracy and efficiency of several radiative transfer models in regard to their applicability to the retrieval of cloud parameters from EPIC measurements. The radiative transfer models, relying on the matrix exponential formalism and endowed with acceleration techniques, include the exact discrete ordinate and matrix operator methods, as well as the approximate asymptotic and equivalent Lambertian cloud models. By "exact" methods, we mean those models used as the starting point to design approximate models by imposing further assumptions.

The paper is organized as follows. In Section 2 we present the exact and approximate radiative transfer models. In Section 3 we summarize the acceleration techniques, focusing on the description of a combined method incorporating the correlated $k$-distribution and PCA. In Section 4 we analyze the accuracy and efficiency of the radiative transfer models for simulated EPIC measurements in the oxygen A-band absorption channel. Conclusions are formulated in Section 5. Some specific features of the radiative transfer models are outlined in the appendices. Here, we give the main computation formulas of the discrete ordinate and matrix operator methods with matrix exponential, justify the equivalent Lambertian cloud model, and describe the telescoping technique.

\section{Radiative transfer models}

The radiative transfer equation for the diffuse radiance $I(r, \mathbf{\Omega})$ at point $r$ and in the direction $\mathbf{\Omega}=(\mu, \varphi)$ reads as

$$
\begin{aligned}
\mu \frac{\mathrm{d} I}{\mathrm{~d} r}(r, \boldsymbol{\Omega}) & =-\sigma_{\mathrm{ext}}(r) I(r, \boldsymbol{\Omega})+F_{0} \frac{\sigma_{\mathrm{sct}}(r)}{4 \pi} P\left(r, \boldsymbol{\Omega}, \boldsymbol{\Omega}_{0}\right) \mathrm{e}^{-\tau_{\mathrm{ext}}^{0}\left(\left|\mathbf{r}-\mathbf{r}_{\mathrm{TOA}}\right|\right)} \\
& +\frac{\sigma_{\mathrm{sct}}(r)}{4 \pi} \int_{4 \pi} P\left(r, \boldsymbol{\Omega}, \boldsymbol{\Omega}^{\prime}\right) I\left(r, \boldsymbol{\Omega}^{\prime}\right) \mathrm{d} \Omega^{\prime}
\end{aligned}
$$

where $\sigma_{\text {ext }}$ and $\sigma_{\text {sct }}$ are the extinction and scattering coefficients, respectively, $F_{0}$ is the incident solar flux, $P$ the scattering phase function, $\boldsymbol{\Omega}_{0}=\left(-\mu_{0}, \varphi_{0}\right)$ with $\mu_{0}>0$ the incident solar direction, and $\tau_{\text {ext }}^{0}\left(\left|\mathbf{r}-\mathbf{r}_{\mathrm{TOA}}\right|\right)$ the solar optical depth between a generic point $\mathbf{r}$ and the characteristic point at the top of the atmosphere $\mathbf{r}_{\mathrm{TOA}}$ in a spherical atmosphere. The formalism is pseudo-spherical, i.e. the multiple-scattering is treated in a plane-parallel atmosphere, while the solar-beam attenuation is computed in a spherical atmosphere [27]. For the phase function $P$, we consider the conventional expansion in terms of normalized Legendre polynomials $P_{n}$, i.e.

$$
P\left(r, \boldsymbol{\Omega}, \mathbf{\Omega}^{\prime}\right)=P(r, \cos \Theta)=\sum_{n=0}^{\infty} c_{n} \chi_{n}(r) P_{n}(\cos \Theta),
$$

where $c_{n}=\sqrt{(2 n+1) / 2}$ and $\cos \Theta=\boldsymbol{\Omega} \cdot \boldsymbol{\Omega}^{\prime}$. The radiative transfer equation (1) is subject to the top-of-atmosphere boundary condition $\left(r=r_{\mathrm{TOA}}\right)$,

$$
I\left(r_{\mathrm{TOA}}, \mathbf{\Omega}^{-}\right)=0
$$

and the surface boundary condition $\left(r=r_{\mathrm{s}}\right)$,

$$
\begin{aligned}
I\left(r_{\mathrm{s}}, \boldsymbol{\Omega}^{+}\right) & =F_{0} \frac{A}{\pi} \mu_{0} \rho\left(\mathbf{\Omega}^{+}, \boldsymbol{\Omega}_{0}\right) \mathrm{e}^{-\tau_{\text {ext }}^{0}\left(\left|\mathbf{r}_{\mathrm{s}}-\mathbf{r}_{\mathrm{TOA}}\right|\right)} \\
& +\frac{A}{\pi} \int_{2 \pi} I\left(r_{\mathrm{s}}, \boldsymbol{\Omega}^{-}\right)\left|\mu^{-}\right| \rho\left(\mathbf{\Omega}^{+}, \boldsymbol{\Omega}^{-}\right) \mathrm{d} \Omega^{-},
\end{aligned}
$$

where $A$ and $\rho$ are the surface albedo and the normalized bidirectional reflection function, respectively, and the notations $\mathbf{\Omega}^{+}$and $\mathbf{\Omega}^{-}$stand for upward and downward directions, respectively.

In the discrete ordinate method, we assume a cosineazimuthal expansion of the diffuse radiance $\left(\varphi_{0}=0\right)$,

$$
I(r, \boldsymbol{\Omega})=\sum_{m=0}^{\infty} I_{m}(r, \mu) \cos m \varphi
$$

and for each azimuthal component $I_{m}(r, \mu)$ we discretize the radiative transfer equation in the angular domain by considering a set of Gauss-Legendre quadrature points and weights $\left\{\mu_{k}, w_{k}\right\}_{k=1}^{M}$ in the interval $(0,1)$; thus, $M$ is the number of discrete ordinates per hemisphere. The atmosphere is discretized in $N$ levels: $r_{1}=r_{\mathrm{TOA}}>r_{2}>\ldots>r_{N}=r_{\mathrm{s}}$, and a layer $j$, bounded above by the level $r_{j}$ and below by the level $r_{j+1}$, has the geometrical thickness $\Delta r_{j}=r_{j}-r_{j+1}$. The extinction and scattering coefficients as well as the phase function coefficients are assumed to be constant within each layer; their average values in layer $j$ are $\sigma_{\text {ext } j}, \sigma_{\text {sct } j}$ and $\chi_{n j}$, respectively. Also, we must require the intensity to be continuous across the layer interfaces. In layer $j$, we are led to the linear system of differential equations

$$
\frac{\mathrm{d} \mathbf{i}_{m}}{\mathrm{~d} r}(r)=\mathbf{A}_{m j} \mathbf{i}_{m}(r)+\mathrm{e}^{-\tau_{\mathrm{ext}}^{0}\left(\left|\mathbf{r}-\mathbf{r}_{\mathrm{TOA}}\right|\right)} \mathbf{b}_{m j}, \quad r_{j+1} \leq r \leq r_{j},
$$

where (the abbreviation "not" stands for a notation definition)

$$
\mathbf{i}_{m}(r)=\left[\begin{array}{c}
\mathbf{i}_{m}^{+}(r) \\
\mathbf{i}_{m}^{-}(r)
\end{array}\right] \stackrel{\text { not }}{=}\left[\mathbf{i}_{m}^{+}(r) ; \mathbf{i}_{m}^{-}(r)\right]^{\top}
$$




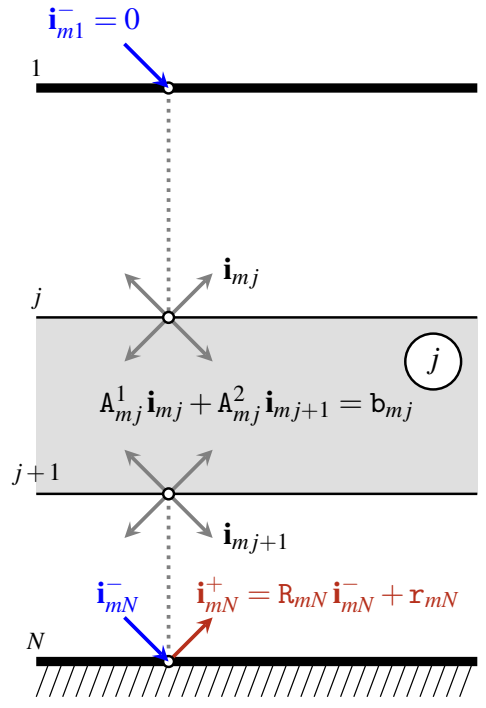

a)

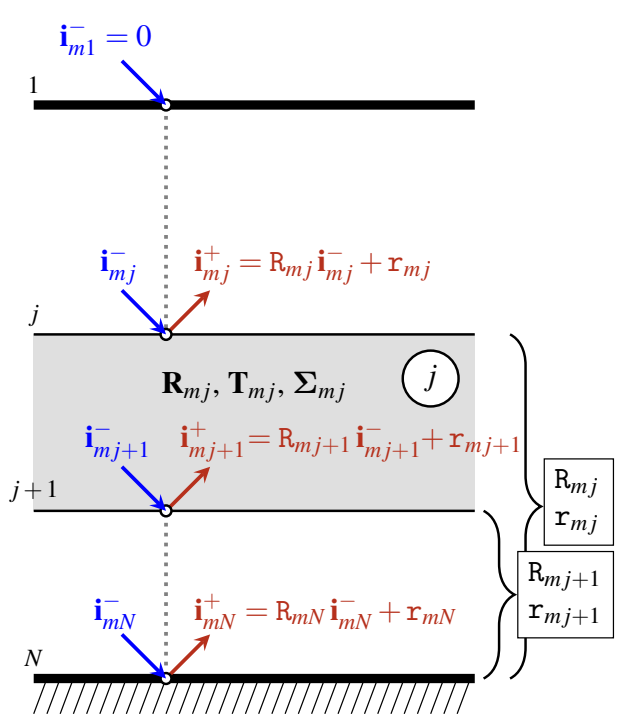

b)

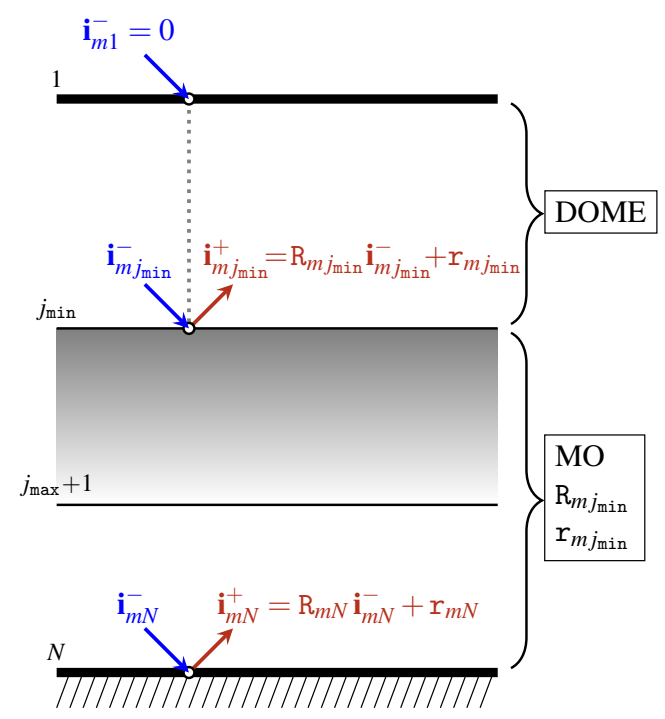

c)

Figure 2: Illustration of DOME (a), the conventional matrix operator method (b), and MOME (c).

is the radiance vector in the discrete-ordinate space, and $\left[\mathbf{i}_{m}^{ \pm}(r)\right]_{k}=I_{m}\left(r, \pm \mu_{k}\right), k=1, \ldots, M$. The layer matrix $\mathbf{A}_{m j}$ has a block structure

$$
\mathbf{A}_{m j}=\left[\begin{array}{cc}
\mathbf{A}_{m j}^{11} & \mathbf{A}_{m j}^{12} \\
-\mathbf{A}_{m j}^{12} & -\mathbf{A}_{m j}^{11}
\end{array}\right]
$$

with entries

$$
\begin{aligned}
{\left[\mathbf{A}_{m j}^{11}\right]_{k l} } & =\frac{1}{2 \mu_{k}}\left[w_{l} \sigma_{\mathrm{sct} j} p_{m j}\left(\mu_{k}, \mu_{l}\right)-2 \sigma_{\mathrm{ext} j} \delta_{k l}\right], \\
{\left[\mathbf{A}_{m j}^{12}\right]_{k l} } & =\frac{1}{2 \mu_{k}} w_{l} \sigma_{\mathrm{sct} j} p_{m j}\left(\mu_{k},-\mu_{l}\right),
\end{aligned}
$$

while the entries of the layer vector $\mathbf{b}_{m j}=\left[\mathbf{b}_{m j}^{+} ; \mathbf{b}_{m j}^{-}\right]^{\top}$ are given by

$$
\left[\mathbf{b}_{m j}^{ \pm}\right]_{k}= \pm \frac{1}{\mu_{k}}\left(2-\delta_{m 0}\right) \frac{F_{0}}{4 \pi} \sigma_{\mathrm{sct} j} p_{m j}\left( \pm \mu_{k},-\mu_{0}\right)
$$

where

$$
p_{m j}\left(\mu, \mu^{\prime}\right)=\sum_{n=m}^{2 M-1} \chi_{n j} P_{n}^{m}(\mu) P_{n}^{m}\left(\mu^{\prime}\right)
$$

are the azimuthal expansion coefficients of the phase functions.

In the following we describe the exact and approximate radiative transfer models for the retrieval of cloud parameters.

\subsection{Discrete ordinate method with matrix exponential}

The Discrete Ordinate method with Matrix Exponential (DOME) [20, 28] is illustrated in Fig. 2a. The method relies on the layer equation, which relates the level values of the radiance field $\mathbf{i}_{m j}=\left[\mathbf{i}_{m j}^{+} ; \mathbf{i}_{m j}^{-}\right]^{\top}$ with $\left[\mathbf{i}_{m j}^{ \pm}\right]_{k}=I_{m}\left(r_{j}, \pm \mu_{k}\right), k=1, \ldots, M$, that is,

$$
\mathrm{A}_{m j}^{1} \mathbf{i}_{m j}+\mathrm{A}_{m j}^{2} \mathbf{i}_{m j+1}=\mathrm{b}_{m j}
$$

The layer equation together with the boundary conditions at the top and the bottom of the atmosphere, i.e. (cf. (3))

$$
\mathbf{i}_{m 1}^{-}=0,
$$

and (cf. (4))

$$
\mathbf{i}_{m N}^{+}=\mathrm{R}_{m N} \mathbf{i}_{m N}^{-}+\mathrm{r}_{m N},
$$

with

$$
\begin{aligned}
{\left[\mathrm{R}_{m N}\right]_{k l} } & =2 A w_{l} \mu_{l} \rho_{m}\left(\mu_{k},-\mu_{l}\right), \\
{\left[\mathrm{r}_{m N}\right]_{k} } & =A \frac{F_{0}}{\pi} \mu_{0} \rho_{m}\left(\mu_{k},-\mu_{0}\right) \mathrm{e}^{-\tau_{\text {ext }}^{0}\left(\left|\mathbf{r}_{\mathrm{s}}-\mathbf{r}_{\mathrm{TOA}}\right|\right),}
\end{aligned}
$$

respectively, are assembled into the global matrix $\mathrm{A}_{m}$ of the entire atmosphere, and the solution of the resulting system of equations $\mathrm{A}_{m} \mathbf{i}_{m}=\mathrm{b}_{m}$ yields the level values of the radiance field. In (16) and (17), $\rho_{m}$ are the azimuthal expansion coefficients of the normalized bi-directional reflection function, i.e.

$$
\rho\left(\mathbf{\Omega}^{+}, \mathbf{\Omega}^{-}\right)=\sum_{m=0}^{2 M-1}\left(2-\delta_{m 0}\right) \rho_{m}\left(\mu,-\mu^{\prime}\right) \cos \left[m\left(\varphi-\varphi^{\prime}\right)\right],
$$

for $\boldsymbol{\Omega}=(\mu, \varphi)$ and $\boldsymbol{\Omega}^{\prime}=\left(-\mu^{\prime}, \varphi^{\prime}\right)$ with $\mu, \mu^{\prime}>0$. The matrix $\mathrm{A}_{m}$ of dimension $2 M N \times 2 M N$ has $3 M-1$ sub- and super-diagonals and it may be compressed into band-storage and then inverted using, for example, the LU factorization.

The layer quantities $\mathrm{A}_{m j}^{1}, \mathrm{~A}_{m j}^{2}$ and $\mathrm{b}_{m j}$ are expressed in terms of the exponential of the layer matrix $\mathbf{A}_{m j}$, i.e. $\exp \left(-\mathbf{A}_{m j} \Delta \boldsymbol{r}_{j}\right)$. The matrix exponential can be computed by using the eigendecomposition method or the Padé approximation; the resulting expressions for $\mathrm{A}_{m j}^{1}, \mathrm{~A}_{m j}^{2}$ and $\mathrm{b}_{m j}$ are listed in Appendix A. The Padé approximation to the matrix exponential is less timeconsuming than the eigendecomposition method, but it is only applicable to optically thin layers, for which the condition $\left\|\mathbf{A}_{m j} \Delta r_{j}\right\| \leq 1$ is satisfied. In practice, if $\left\|\mathbf{A}_{m j} \Delta r_{j}\right\|_{1} \leq 1$, the matrix exponential is computed by means of the Padé approximation; otherwise, the eigendecomposition method is used. By this procedure, the computation speed is enhanced. 


\subsection{Matrix operator method with matrix exponential}

The Matrix Operator method with Matrix Exponential (MOME) is a combination of the conventional matrix operator method, e.g. [21], and the discrete ordinate method with matrix exponential. The method is dedicated to modeling the radiative transfer in a molecular atmosphere containing a homogeneous cloud placed between the top level $r_{j_{\min }}$ and the bottom level $r_{j_{\max }+1}$. Note that the cloud homogeneity assumption is only required by the approximate models (in particular, by the asymptotic model) to be introduced in the next subsection.

Before describing MOME, we briefly review the basic concepts of the conventional matrix operator method. The method, which is illustrated in Fig. $2 \mathrm{~b}$, uses the interaction principle equation

$$
\left[\begin{array}{c}
\mathbf{i}_{m j}^{+} \\
\mathbf{i}_{m j+1}^{-}
\end{array}\right]=\left[\begin{array}{cc}
\mathbf{R}_{m j} & \mathbf{T}_{m j} \\
\mathbf{T}_{m j} & \mathbf{R}_{m j}
\end{array}\right]\left[\begin{array}{c}
\mathbf{i}_{m j}^{-} \\
\mathbf{i}_{m j+1}^{+}
\end{array}\right]+\left[\begin{array}{c}
\boldsymbol{\Sigma}_{m j}^{+} \\
\boldsymbol{\Sigma}_{m j}^{-}
\end{array}\right],
$$

where $\mathbf{R}_{m j}$ and $\mathbf{T}_{m j}$ are the reflection and transmission matrices of layer $j$, and $\boldsymbol{\Sigma}_{m j}^{ \pm}$is the source vector. As in DOME, $\mathbf{R}_{m j}, \mathbf{T}_{m j}$ and $\boldsymbol{\Sigma}_{m j}$ are given in terms of the exponential of the layer matrix $\mathbf{A}_{m j}$; their expressions computed by using the matrix eigendecomposition method and the Padé approximation are given in Appendix B. The computation process is an upward recurrence over the atmospheric layers, and uses the concept of a "stack" [29]. The stack $j+1$, i.e. the group of contiguous layers bounded above by the level $r_{j+1}$ and below by the surface level $r_{N}$, is characterized by the reflection matrix $\mathrm{R}_{m j+1}$ and the reflection vector $\mathbf{r}_{m j+1}$, so that the interaction principle equation for the stack $j+1$ reads as

$$
\mathbf{i}_{m j+1}^{+}=\mathrm{R}_{m j+1} \mathbf{i}_{m j+1}^{-}+\mathbf{r}_{m j+1} .
$$

From (19) and (20), we obtain the interaction principle equation for stack $j$, i.e. the group of contiguous layers bounded above by the level $r_{j}$ and below by the surface level $r_{N}$,

$$
\mathbf{i}_{m j}^{+}=\mathrm{R}_{m j} \mathbf{i}_{m j}^{-}+\mathrm{r}_{m j},
$$

where $\mathrm{R}_{m j}$ and $\mathrm{r}_{m j}$ are computed by using the adding formulas

$$
\begin{aligned}
\mathbf{R}_{m j} & =\mathbf{R}_{m j}+\mathbf{T}_{m j} \mathbf{R}_{m j+1}\left(\mathbf{I}-\mathbf{R}_{m j} \mathbf{R}_{m j+1}\right)^{-1} \mathbf{T}_{m j}, \\
\mathbf{r}_{m j} & =\Sigma_{m j}^{+}+\mathbf{T}_{m j} r_{m j+1} \\
& +\mathbf{T}_{m j} \mathbf{R}_{m j+1}\left(\mathbf{I}-\mathbf{R}_{m j} \mathbf{R}_{m j+1}\right)^{-1}\left(\mathbf{R}_{m j} \mathbf{r}_{m j+1}+\Sigma_{m j}^{-}\right) .
\end{aligned}
$$

The procedure is initialized with (15) and it is repeated until the last layer is added to the stack. In the matrix operator method, the dimension of the problem is small, because the dimension of the matrices that have to be inverted is $2 M \times 2 M$. In spite of this fact, its efficiency is reduced because of the considerable number of matrix inversions and matrix multiplications required by the adding algorithm. Another disadvantage is that the computation of the radiance in an arbitrary viewing direction by the source function integration method requires the storage of the reflection and transmission matrices of all individual layers. Alternatively, and to keep the memory usage low, we may interpolate the radiance field in the discrete ordinate space by cubic splines, or we may use the method of false discrete ordinate (see Section 2.3).

The combined model MOME, which is illustrated in Fig. 2c, involves two computation steps:

1. compute the reflection matrix $\mathrm{R}_{m j_{\min }}$ and the reflection vector $\mathrm{r}_{m j_{\min }}$ of stack $j_{\text {min }}$ (containing all layers below the cloud top height) in the framework of the conventional matrix operator method;

2. compute the level values of the radiance $\mathbf{i}_{m j}$ for $1 \leq j \leq j_{\min }$, in the framework of the discrete ordinate method with matrix exponential, by using as surface boundary condition the interaction principle equation

$$
\mathbf{i}_{m j_{\min }}^{+}=\mathrm{R}_{m j_{\min }} \mathbf{i}_{m j_{\min }}^{-}+\mathrm{r}_{m j_{\min }}
$$

Taking this model as a starting point, several approximate and less time-consuming models have been designed. These are described in the next subsection.

\subsection{Approximate models}

The approximate models are based on some preliminary simplifications which we now describe. Firstly, the atmosphere below the cloud bottom height is replaced by an equivalent Lambertian surface. The resulting model corresponding to a cloud with an underlying Lambertian surface can be summarized as follows:

1. for the azimuthal mode $m=0$, compute the reflection matrix $\mathrm{R}_{m j_{\max }+1}$ and the reflection vector $\mathrm{r}_{m j_{\max }+1}$ of stack $j_{\max }$ containing all layers below the cloud bottom height;

2. compute the spherical albedo at the cloud bottom

$$
A_{\mathrm{cb}}=2 \mathbf{v}^{\top} \mathrm{R}_{m j_{\max }+1} \mathbf{1}
$$

where $[\mathbf{v}]_{k}=w_{k} \mu_{k}, k=1, \ldots, M$ and $\mathbf{1}=[1, \ldots, 1]^{\top}$;

3. use the surface boundary condition

$$
\mathbf{i}_{m j_{\max }+1}^{+}=\mathrm{R}_{A_{\mathrm{cb}}} \mathbf{i}_{m j_{\max }+1}^{-}+\mathrm{r}_{A_{\mathrm{cb}}} \text { for } m=0,
$$

with

$$
\begin{aligned}
{\left[\mathrm{R}_{A_{\mathrm{cb}}}\right]_{k l} } & =2 A_{\mathrm{cb}} w_{l} \mu_{l}, \\
{\left[\mathrm{r}_{A_{\mathrm{cb}}}\right]_{k} } & =A_{\mathrm{cb}} \frac{F_{0}}{\pi} \mu_{0} \mathrm{e}^{-\tau_{\mathrm{ext}}^{0}\left(\left|\mathbf{r}_{\mathrm{cb}}-\mathbf{r}_{\mathrm{TOA}}\right|\right)},
\end{aligned}
$$

to initialize the adding procedure for computing the reflection matrix $\mathrm{R}_{m j_{\min }}$ and the reflection vector $\mathrm{r}_{m j_{\min }}$ of stack $j_{\text {min }}$ containing all layers below the cloud top height;

4. compute the radiance field of the atmosphere above the cloud top height using as surface boundary condition the interaction principle equation (24).

Secondly, the atmosphere within the cloud (between the layers $j_{\min }$ and $\left.j_{\max }\right)$ is homogenized. The equivalent homogeneous layer $j_{\min }$ is characterized by

$$
\sigma_{\mathrm{ext} j_{\min }}^{\text {hom }}=\frac{\sum_{j=j_{\min }}^{j_{\max }} \sigma_{\mathrm{ext} j} \Delta r_{j}}{\sum_{j=j_{\min }}^{j_{\max }} \Delta r_{j}}, \quad \sigma_{\mathrm{sct} j_{\min }}^{\text {hom }}=\frac{\sum_{j=j_{\min }}^{j_{\max }} \sigma_{\mathrm{sct} j} \Delta r_{j}}{\sum_{j=j_{\min }}^{j_{\max }} \Delta r_{j}},
$$




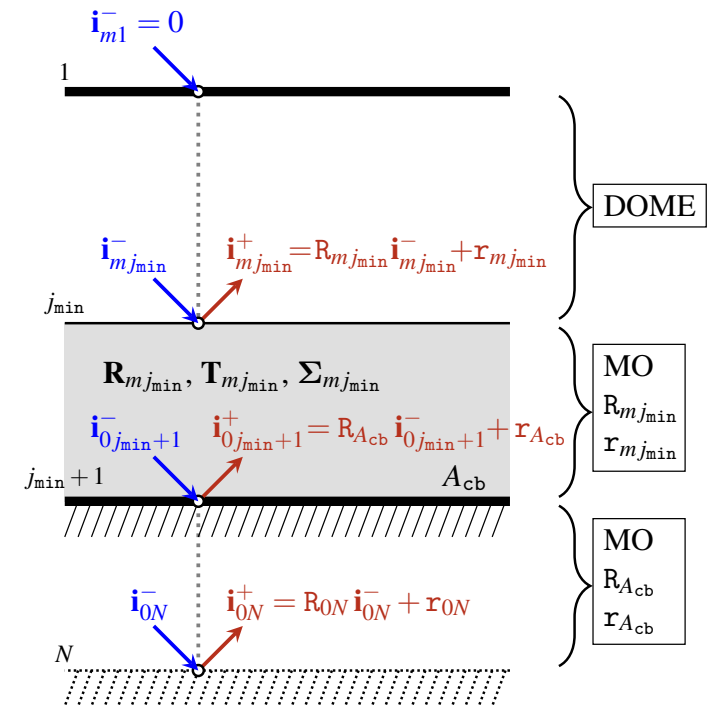

a)

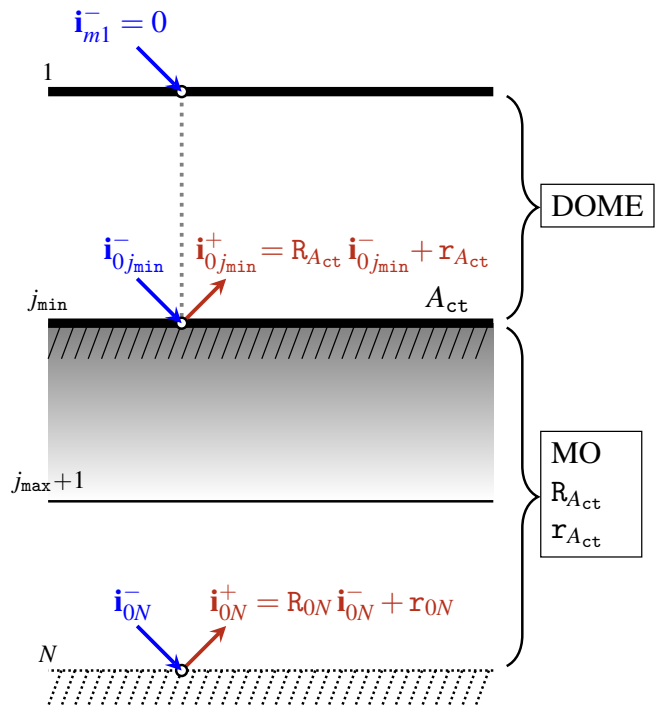

b)

Figure 3: Illustration of a homogeneous cloud layer with an underlying Lambertian surface (a) and of an equivalent Lambertian cloud (b).

and

$$
\sigma_{\mathrm{sct} j_{\min }}^{\text {hom }} \chi_{n j_{\min }}^{\text {hom }}=\frac{\sum_{j=j_{\min }}^{j_{\max }} \sigma_{\mathrm{sct} j} \chi_{n j} \Delta r_{j}}{\sum_{j=j_{\min }}^{j_{\max }} \Delta r_{j}}, \quad n=m, \ldots, 2 M-1
$$

The resulting model, corresponding to a homogeneous cloud layer with an underlying Lambertian surface and being illustrated in Fig. 3a, requires only one adding step. The homogenization accounts for the scattering and absorption in the cloud, the Rayleigh scattering, as well as the gas absorption between the layers $j_{\min }$ and $j_{\max }$. Strictly speaking, the equivalent homogeneous extinction coefficient $\sigma_{\text {ext } j_{\min }}^{\text {hom }}$ should be computed, for example, by minimizing the mismatch between the radiance corresponding to an inhomogeneous atmosphere within the cloud and the radiance corresponding to a homogeneous atmosphere. Although the homogenization formula (29) is a potential source of error (especially for strong gas absorption), it has been adopted in our analysis because of its simplicity.

We come now to the first approximate model. When the optical thickness is sufficiently large, the reflection and transmission matrices $\mathbf{R}_{m j_{\min }}$ and $\mathbf{T}_{m j_{\min }}$ in (22) and (23) can be expressed by simple analytical expressions deriving from the asymptotic theory of thick layers. In the classical asymptotic theory, the reflection and transmission matrices for optically thick layers are given by (we omit the dependency on the layer index $j_{\min }$ ) [30]

$$
\begin{aligned}
& \mathbb{R}_{m}=\mathbb{R}_{\infty m}-\delta_{m 0} \frac{\mathrm{m} l \mathrm{e}^{-2 \mathrm{k} \tau}}{1-1^{2} \mathrm{e}^{-2 \mathrm{k} \tau}} \mathbf{k}_{m M} \mathbf{k}_{m M}^{\top}, \\
& \mathbb{T}_{m}=\delta_{m 0} \frac{\mathrm{m} \mathrm{e}^{-\mathrm{k} \tau}}{1-1^{2} \mathrm{e}^{-2 \mathrm{k} \tau}} \mathbf{k}_{m M} \mathbf{k}_{m M}^{\top},
\end{aligned}
$$

where $\mathbb{R}_{m}=(1 / 2) \mathbf{R}_{m} \mathbf{M} \mathbf{W}^{-1}$ and $\mathbb{T}_{m}=(1 / 2) \mathbf{T}_{m} \mathbf{M} \mathbf{W}^{-1}$ are the discrete-ordinate approximations of the reflection and transmission functions of the homogeneous layer $R_{m}\left(\mu, \mu^{\prime}\right)$ and $T_{m}\left(\mu, \mu^{\prime}\right)$, respectively, $\mathbb{R}_{\infty m}=(1 / 2) \mathbf{R}_{\infty m} \mathbf{M} \mathbf{W}^{-1}$ is the discrete- ordinate approximation of the reflection function of a semi-infinite atmosphere $R_{\infty m}\left(\mu, \mu^{\prime}\right),[\mathbf{W}]_{k l}=w_{k} \delta_{k l}$, and $[\mathbf{M}]_{k l}=\left(1 / \mu_{k}\right) \delta_{k l}$, $k, l=1, \ldots, M$. Furthermore, in (31) and (32), $\mathrm{k}=\lambda_{M}$ is the diffusion exponent describing the attenuation of the radiation in the diffusion domain, $\lambda_{M}$ is the smallest eigenvalue of the layer matrix $\mathbf{A}_{m}, \tau$ is the optical thickness of the homogeneous layer, $m$ and 1 are the constants defined in [30], and $\mathbf{k}_{0 M}$ is a discrete-ordinate approximation of the escape function $K(\mu)$. From (31) and (32) we see that $\mathbb{R}_{m}$ depends on the azimuthal index $m$ through the reflection matrix of a semi-infinite atmosphere $\mathbb{R}_{\infty m}$, while $\mathbb{T}_{m}$ is azimuthally independent (it depends only on the azimuthal mode $m=0$ ). A discrete-ordinate model for computing $\mathbb{R}_{m}$ and $\mathbb{T}_{m}$, and so $\mathbf{R}_{m}$ and $\mathbf{T}_{m}$, is described in [31]. In this model, the azimuthal independent parts of $\mathbb{R}_{m}$ and $\mathbb{T}_{m}$ are derived by neglecting the azimuthal modes $m>0$, and the contributions of the terms corresponding to eigenvalues larger than the smallest eigenvalue $\lambda_{M}$ of $\mathbf{A}_{m}$. A more accurate asymptotic model, the so-called $P$-order asymptotic model, has been introduced in [32]. This model accounts for all azimuthal modes $m$, and the contributions of the terms corresponding to eigenvalues

$$
\lambda_{M-P} \geq \lambda_{M-(P-1)} \geq \cdots \geq \lambda_{M}
$$

where $P$ is the order of approximation. The case $P=0$ corresponds in some sense to the classical asymptotic model, as it accounts for all azimuthal modes $m$. It should be pointed out that, in the classical asymptotic theory, the computation of the reflection and transmission matrices can be speeded up by using parametrizations of the constants and functions appearing in (31) and (32) $[32,33]$. In our numerical analysis we are mainly concerned about the accuracy of the approximate models and, for this reason, we do not use any parametrization in (31) and (32); we simulate a $P$-order asymptotic model by simply setting

$$
\Gamma_{m j}=\operatorname{diag}\left[0, \ldots, 0, \exp \left(-\lambda_{M-P} \Delta r_{j}\right), \ldots, \exp \left(-\lambda_{M} \Delta r_{j}\right)\right]
$$

in place of (60) in Appendix B. 
The second approximate model to be discussed is the Equivalent Lambertian Cloud (ELC) model (see Fig. 3b). In the ELC model, the atmosphere below the cloud top height is replaced by an equivalent Lambertian surface with a ground albedo equal to the spherical albedo of the atmosphere. The mathematical background of the ELC model is discussed in Appendix C. In the framework of MOME, this model can be simulated as follows:

1. for the azimuthal mode $m=0$, compute the reflection matrix $\mathrm{R}_{m j_{\min }}$ and the reflection vector $\mathrm{r}_{m j_{\min }}$ of stack $j_{\text {min }}$ containing all layers below the cloud top height;

2. compute the spherical albedo at the cloud top

$$
A_{\mathrm{ct}}=2 \mathbf{v}^{\top} \mathrm{R}_{m j_{\min }} \mathbf{1}
$$

3. compute the radiance field of the atmosphere above the cloud top height by using the surface boundary condition

$$
\mathbf{i}_{m j_{\min }}^{+}=\mathrm{R}_{A_{\mathrm{ct}}} \mathbf{i}_{m j_{\min }}^{-}+\mathrm{r}_{A_{\mathrm{ct}}} \quad \text { for } m=0 .
$$

The ELC model ensures the conservation of radiative flux if the plane albedo $r(\mu)$ is almost constant with respect to $\mu$. Note that in a discrete-ordinate setting, the plane albedo $r(\mu)$ is modeled by the plane albedo vector $\mathbf{r}=\mathrm{R}_{m j_{\min }} \mathbf{1}$.

\section{Acceleration techniques}

In addition to the Padé approximation, the standard delta-M method [11], which expands the original phase function into a delta-function forward peak plus a new less anisotropic phase function, and the truncated-plus-single-scattering (TMS) method [34], several acceleration techniques are implemented in the radiative transfer models. These are summarized below.

\subsection{The telescoping technique}

The telescoping technique relies on the following result [19, 20]: For an atmosphere consisting of gas molecules (Rayleigh layers) and a group of contiguous cloud layers, the azimuthal expansion coefficients of the phase functions $p_{m j}$ vanish for all $m>2$, and all Rayleigh layers $j$. Consequently, the layer matrix $\mathbf{A}_{m j}$ becomes a diagonal matrix and the layer vector $\mathbf{b}_{m j}$ vanishes. The main idea of the telescoping technique is to solve a reduced boundary-value problem for the cloud layers, and to compute the radiances at the remaining levels recursively by means of the extinction law. The telescoping technique for DOME and MOME is outlined in Appendix D.

\subsection{The method of false discrete ordinate}

The method of false discrete ordinate has been discussed in connection with the matrix operator method in [21-23] and the discrete ordinate method with matrix exponential in [24]. For a single viewing direction, an additional stream (directional cosine) is introduced as an extra Gaussian quadrature point with zero weight. The upward radiance at the false discrete ordinate is exactly the upward radiance in the direction of the line of sight computed by the source function integration method. The method of false discrete ordinate eliminates the source function integration step, but increases the dimension of the layer equation. As a result, for applications involving one or two viewing zenith angles, the method of false discrete ordinate does not increase significantly the computation time of the forward model.

\subsection{The correlated $k$-distribution method and the PCA tech- nique}

The correlated $k$-distribution method [10] involves grouping spectral intervals according to absorption coefficient strength, while PCA is used here as a dimensionality reduction technique of the optical properties [16-18]. Although these methods have been applied separately in the literature, they can work together. To show this, we briefly summarize their basic concepts.

Let $g(\lambda)$ be the slit function of the instrument, $s$ the slit width, and $\left\{\lambda_{k}\right\}_{k=1}^{N_{\lambda}}$ a discrete set of $N_{\lambda}$ equally-spaced wavelengths in the interval $[\lambda-s / 2, \lambda+s / 2]$ with

$$
\lambda_{1}-\frac{\Delta \lambda}{2}=\lambda-\frac{s}{2} \quad \text { and } \quad \lambda_{N_{\lambda}}+\frac{\Delta \lambda}{2}=\lambda+\frac{s}{2},
$$

where $\Delta \lambda$ is the discretization step. The signal received by the instrument is the convolution of the slit function and the monochromatic radiance, and can be approximated by

$$
\begin{aligned}
I(\lambda) & =\int_{\lambda-s / 2}^{\lambda+s / 2} g\left(\lambda-\lambda^{\prime}\right) I\left(\lambda^{\prime}\right) \mathrm{d} \lambda^{\prime} \\
& \approx \sum_{k=1}^{N_{\lambda}} g\left(\lambda-\lambda_{k}\right) \int_{\lambda_{k}-\Delta \lambda / 2}^{\lambda_{k}+\Delta \lambda / 2} I\left(\lambda^{\prime}\right) \mathrm{d} \lambda^{\prime} .
\end{aligned}
$$

As gas absorption has greater spectral variation than molecular and particulate scattering, we may write $I(\lambda)=I\left(\sigma_{\mathrm{abs}}^{\mathrm{gas}}(\lambda)\right)$. The most accurate method for computing the integral in (33) involves a detailed line-by-line (LBL) calculation of the gas absorption coefficient versus wavelength. On the other hand, the correlated $k$-distribution method is based on the observation that, for a homogeneous atmosphere, the transmission within a spectral interval is independent of the LBL variation of the gas absorption coefficient $\sigma_{\text {abs }}^{\text {gas }}$ with respect to the wavelength $\lambda$, but depends only on the distribution of $\sigma_{\text {abs }}^{\text {gas }}$ within the interval [35]. In this regard, let $F=F\left(\sigma_{\text {absk }}^{\text {gas }}\right)$ be the cumulative density function of $\sigma_{\text {abs }}^{\text {gas }}(\lambda)$ in the spectral interval $\left[\lambda_{k}-\Delta \lambda / 2, \lambda_{k}+\Delta \lambda / 2\right]$, and $\sigma_{\text {abs } k}^{\text {gas }}(F)$ the quantile function or the inverse distribution function. The signal received by the instrument can then be computed as

$$
\begin{aligned}
I(\lambda) & =\Delta \lambda \sum_{k=1}^{N_{\lambda}} g\left(\lambda-\lambda_{k}\right) \int_{0}^{1} I\left(\sigma_{\mathrm{abs} k}^{\mathrm{gas}}(F)\right) \mathrm{d} F \\
& =\Delta \lambda \sum_{k=1}^{N_{\lambda}} g\left(\lambda-\lambda_{k}\right) \sum_{l=1}^{N_{\mathrm{c}}} \omega_{l} I\left(\sigma_{\mathrm{abs} k}^{\mathrm{gas}}\left(F_{l}\right)\right),
\end{aligned}
$$

where $\left(F_{l}, \omega_{l}\right)$ are a set of $N_{\mathrm{c}}$ quadrature points and weights within the interval $[0,1]$. The $\sigma_{\text {absk }}^{\text {gas }}\left(F_{l}\right)$ can be computed by inverting the cumulative density functions of the LBL gas absorption coefficients or, in the case of the "exponential-sum 
fitting of transmittance" method [11], by solving a nonlinear least squares problem of dimension $N_{\mathrm{c}} \times M$. Let us define a new set of wavelengths $\left\{\bar{\lambda}_{p}\right\}_{p=1}^{N_{\bar{\lambda}}}$ and weights $\left\{\bar{\omega}_{p}\right\}_{p=1}^{N_{\bar{\lambda}}}$ with $N_{\bar{\lambda}}=N_{\lambda} N_{\mathrm{c}}$, through the relations $\bar{\lambda}_{p}=\lambda_{k}$ and $\bar{\omega}_{p}=\Delta \lambda \omega_{l}$, where $p=l+(k-1) N_{\mathrm{c}}$ for $k=1, \ldots, N_{\lambda}, l=1, \ldots, N_{\mathrm{c}}$, and set accordingly $\sigma_{\text {abs }}^{\text {gas }}\left(\bar{\lambda}_{p}\right)=\sigma_{\text {abs } k}^{\text {gas }}\left(F_{l}\right)$. Note that $\left\{\bar{\lambda}_{p}\right\}_{p=1}^{N_{\bar{\lambda}}}$ contains $N_{\lambda}$ groups of $N_{\mathrm{c}}$ identical wavelengths. By this construction, (34) becomes

$$
I(\lambda)=\sum_{p=1}^{N_{\bar{\lambda}}} \bar{\omega}_{p} g\left(\lambda-\bar{\lambda}_{p}\right) I\left(\sigma_{\mathrm{abs}}^{\mathrm{gas}}\left(\bar{\lambda}_{p}\right)\right),
$$

and it is apparent that (35) is a quadrature rule for (33) in the case of the correlated $k$-distribution method.

We come now to the PCA technique. A general approximate model for computing the radiance $I$ at the wavelength $\lambda$ is of the form

$$
\ln \frac{I(\lambda)}{I_{\mathrm{a}}(\lambda)}=f_{\mathrm{I}}(\lambda)
$$

where $I_{\mathrm{a}}$ is the radiance computed by an approximate radiative transfer model, and $f_{\mathrm{I}}$ is a correction factor. An efficient and accurate method for computing the radiance correction factor $f_{\mathrm{I}}(\lambda)$ has been given by Natraj et al. $[16,17]$. This approach, which increases the computational efficiency of the radiative transfer calculations in an absorbing and scattering atmosphere, has the following attributes:

1. The exact model is the multi-stream DOME model, while the approximate model is a two-stream version of DOME, in which the eigenvalues and the eigenvectors of the layer matrix are computed analytically, and the system of equations for the entire atmosphere is solved by means of a pentadiagonal linear algebra solver.

2. The principal component analysis (PCA) is used to reduce the dimensionality of the optical parameters of the atmospheric system. In particular, PCA is performed on the logarithms of the layer values of the gas absorption coefficient $\ln \sigma_{\text {abs }}^{\text {gas }}(\lambda)$ and the molecular scattering coefficient $\ln \sigma_{\mathrm{sct}}^{\mathrm{mol}}(\lambda)$; for each $\lambda_{k}, k=1, \ldots N_{\lambda}$, we define the $(2 N-2)$-dimensional vector

$$
\mathbf{x}\left(\lambda_{k}\right) \stackrel{\text { not }}{=} \mathbf{x}_{k}=\left[\ln \sigma_{\mathrm{abs} j}^{\mathrm{gas}}\left(\lambda_{k}\right) ; \ln \sigma_{\mathrm{sct} j}^{\mathrm{mol}}\left(\lambda_{k}\right)\right]^{\mathrm{T}},
$$

$j=1, \ldots, N-1$, so that the wavelength variability of the optical parameters is encapsulated in $\mathbf{x}_{k}$.

3. The dependency of the correction factor on the optical parameters is modeled by a second-order Taylor expansion about the mean value of the optical parameters.

Parenthetically, we note that in [18] the PCA-based radiative transfer model of Natraj et al. [16, 17] has been generalized to include several dimensionality reduction techniques as, for example, linear embedding methods and discrete orthogonal transforms. The PCA formalism remains valid if instead of the set $\left\{\lambda_{k}\right\}_{k=1}^{N_{\lambda}}$ we consider the set $\left\{\bar{\lambda}_{p}\right\}_{p=1}^{N_{\bar{\lambda}}}$ of the correlated $k$-distribution method (the wavelength variability is encapsulated in $\left.\mathbf{x}\left(\bar{\lambda}_{p}\right)\right)$. This claim is checked in the next section.

\section{Numerical simulations}

In this section we analyze the accuracy and efficiency of the exact and approximate radiative transfer models in the oxygen $\left(\mathrm{O}_{2}\right)$ A-band absorption channel at $764 \mathrm{~nm}$ (channel 9 of the EPIC instrument). For this purpose, simulations are performed for a water-cloud model with a Gamma size distribution

$$
P(a) \propto a^{\alpha} \exp \left[-\alpha\left(\frac{a}{a_{\text {mod }}}\right)\right]
$$

with parameters $a_{\text {mod }}=8 \mu \mathrm{m}$ and $\alpha=6$. The droplet size ranges between 0.02 and $50.0 \mu \mathrm{m}$. The cloud top height is $h_{\mathrm{t}}=4 \mathrm{~km}$, and the cloud geometrical thickness $\Delta h$ is chosen as

$$
\Delta h=\left\{\begin{array}{l}
1.0 \mathrm{~km}, \quad \tau_{\mathrm{c}} \leq 4 \\
1.5 \mathrm{~km}, \quad 4<\tau_{\mathrm{c}} \leq 8 \\
2.0 \mathrm{~km}, \quad 8<\tau_{\mathrm{c}} \leq 14 \\
2.5 \mathrm{~km}, \quad 14<\tau_{\mathrm{c}} \leq 20 \\
3.0 \mathrm{~km}, \quad \tau_{\mathrm{c}}>20
\end{array},\right.
$$

where $\tau_{\mathrm{c}}$ is the cloud optical thickness. The atmosphere is discretized with a step of $0.5 \mathrm{~km}$ between 0 and $16 \mathrm{~km}$, a step of $2 \mathrm{~km}$ between $16 \mathrm{~km}$ and $20 \mathrm{~km}$, a step of $5 \mathrm{~km}$ between $20 \mathrm{~km}$ and $30 \mathrm{~km}$, and, finally, a step of $10 \mathrm{~km}$ between $30 \mathrm{~km}$ and $50 \mathrm{~km}$. The ground surface is Lambertian with albedo $A=0.06$, and, as in [3], the solar and viewing zenith angles are taken to be equal, i.e. $\theta=\theta_{0}$, while the relative azimuthal angle between the solar and viewing directions is chosen as $\Delta \varphi=176^{\circ}$. The azimuthal convergence test is the standard DISORT double convergence test [36] with a tolerance of $10^{-6}$. The $\mathrm{O}_{2}$ absorption cross sections are computed by using LBL calculations [37] with optimized rational approximations for the Voigt line profile [38]. The wavenumber grid point spacing is chosen as a fraction (e.g. 1/4) of the minimum half-width of the Voigt lines taken from HITRAN database [39]. The Rayleigh crosssections and depolarization ratios are computed as in [40], while the pressure and temperature profiles correspond to the US standard model atmosphere [41], and this atmosphere is considered free of aerosols. The radiances are solar-flux normalized, and are computed by means of the delta-M approximation in conjunction with the TMS correction. If not stated otherwise the simulations are based on LBL calculations. The instrument spectral response functions (ISRF) for the different EPIC channels are available from NASA public servers. ${ }^{1}$

\subsection{Exact models}

In Fig. 4 we show the DOME radiances as functions of the cloud optical thickness $\tau_{\mathrm{c}}$ and for different values of the viewing zenith angle $\theta$. The simulations are performed by using a large number of discrete ordinates, namely $M=128$. The plots show that, for a fixed value of $\theta$, the radiance is an increasing function of $\tau_{\mathrm{c}}$. The reason is that the multiple-scattering contribution, which is an increasing function of $\tau_{c}$, dominates the

\footnotetext{
${ }^{1}$ https://avdc.gsfc.nasa.gov/pub/DSCOVR/EPIC_Filter_Data/ EPIC_Filters_Original_Data.xlsx.
} 


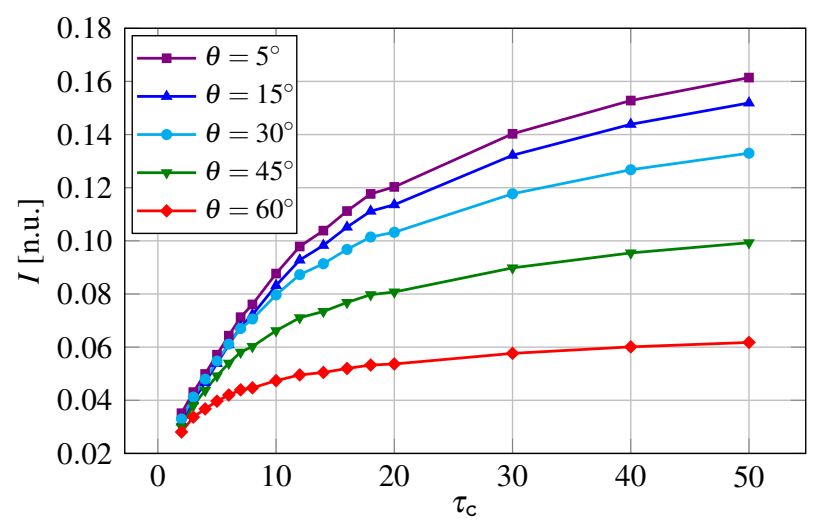

Figure 4: Reference DOME radiances as functions of $\tau_{\mathrm{c}}$ for $M=128$. In the $y$-axis, [n.u.] stands for normalized units.

single-scattering contribution, which is a decreasing function of $\tau_{\mathrm{c}}$. Moreover, for a fixed value of $\tau_{\mathrm{c}}$, the radiances decrease as the viewing zenith angle $\theta$ increases (if $\theta$ increases, the path through the atmosphere increases, the amount of oxygen absorption increases, and so, the single-scattering contribution decreases). These results are taken as the "exact" reference values for the rest of the simulations that follow.

In Fig. 5 we illustrate the relative errors in DOME radiances for different values of the number of discrete ordinates $M$. The plots show that, for a fixed value of $\theta$, the absolute values of the relative errors decrease with $\tau_{\mathrm{c}}$. The reason is that, now, the relative error in the single-scattering contribution dominates the relative error in the multiple-scattering contribution. The results in Fig. 5 can be summarized as follows:

1. for $M=8$ and $M=16$, the relative errors are smaller than 0.015 for $\tau_{\mathrm{c}} \geq 10$, and lie roughly between 0.015 and 0.03 for $\tau_{\mathrm{c}}<10$;

2. for $M=24$ and $M=32$, the relative errors are smaller than 0.008 for $\tau_{c} \geq 10$, and lie between 0.008 and 0.015 for $\tau_{\mathrm{c}}<10$;

3. for $M=48$, the relative errors are smaller than 0.01 over the entire range of $\tau_{\mathrm{c}}$;

4. for $M=64$, the relative errors are smaller than 0.003 over the entire range of $\tau_{\mathrm{c}}$.

The computation times for these simulations are given in Table 2. Note that the simulations were performed on a server Intel(R) Xeon(R) CPU E5-2695 v3 @ 2.30 GHz using up to 56 threads, and that the computation times in Table 2 correspond to the accumulated time over all threads. Thus, even though the delta-M scaling is used, an accurate description of the backscattering region requires $M \geq 48$, although a compromise between speed and accuracy can be reached by the choice $24 \leq M \leq 32$. Note also that for $M \geq 24$ more than 30 azimuthal modes are required to reach convergence with an accuracy of $10^{-6}$.

Fig. 6 illustrates the relative errors in MOME radiances using DOME as a reference. Taking into account that the relative errors are smaller than $5 \cdot 10^{-4}$, and that the MOME computation user time is 1660 minutes for $M=32$, we infer that DOME and MOME yield similar results from the point of view of accuracy and efficiency.
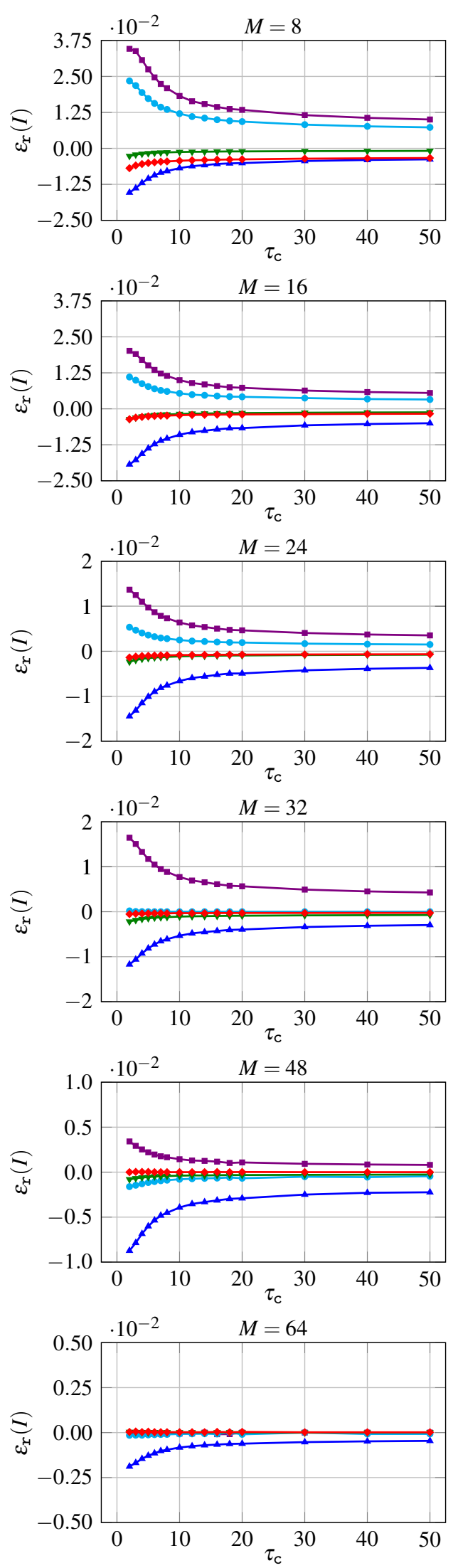

$\varpi \theta=5^{\circ} \multimap \theta=15^{\circ} \multimap \theta=30^{\circ} \multimap-\theta=45^{\circ} \multimap \theta=60^{\circ}$

Figure 5: Relative errors in DOME radiances as functions of $\tau_{\mathrm{c}}$ and for different values of $M$. 
Table 2: Computation user times in minutes for the results in Fig. 5, and speedup factors with respect to the case $M=128$.

\begin{tabular}{rrr}
\hline $\boldsymbol{M}$ & $\mathbf{t} / \mathbf{m i n}$ & Speed-up factor \\
\hline 8 & 67 & 2389.8 \\
16 & 284 & 563.8 \\
24 & 760 & 210.7 \\
32 & 1711 & 93.6 \\
48 & 5698 & 28.1 \\
64 & 14548 & 11.0 \\
128 & 160116 & - \\
\hline
\end{tabular}

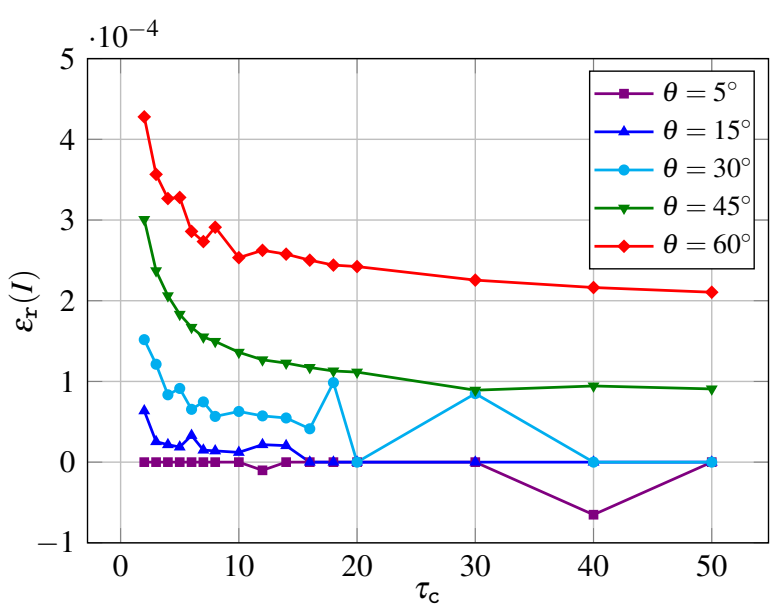

Figure 6: Relative errors in MOME radiances with respect to DOME radiances for $M=32$.

\subsection{Approximate models}

The relative errors of the asymptotic (fourth- and zerothorder) and ELC models are shown in Fig.7. A general finding is that an increase of the number of discrete ordinates does not significantly improve the accuracy of the results; a real improvement is apparent only for the fourth-order asymptotic model. The relative errors of the fourth-order asymptotic model are smaller than 0.02 for $\tau_{\mathrm{c}} \geq 10$, and smaller than 0.04 for $\tau_{\mathrm{c}}<10$. For the zeroth-order asymptotic model, the relative errors have a similar behaviour when $\tau_{\mathrm{c}} \geq 10$, but they can reach 0.24 when $\tau_{\mathrm{c}}<10$.

The relative errors of the ELC model are excessively large; they can reach 0.4 for small $\tau_{\mathrm{c}}$, and are 0.1 for large $\tau_{\mathrm{c}}$. The main reason for this is that the plane albedo $r(\mu)$ varies significantly with $\mu$, and so, the radiative flux is not conserved (Fig.8). Note that, even in the limit of large $\tau_{\mathrm{c}}$, the flux conservation does not imply an accurate description of the radiance field (the radiative flux description involves only the azimuthal mode $m=0$ ). Also note that the spherical albedo $A_{\mathrm{ct}}$ changes with $\tau_{\mathrm{c}}$, a fact which contradicts the common assumption of a constant cloud albedo [9]. The following result is a consequence of the low accuracy of the ELC model. Let $I\left(A_{\mathrm{ct} 0}, h_{\mathrm{t} 0}\right)$ be the radiance computed by an exact model corresponding to a cloud with top height $h_{\mathrm{t} 0}$ and a spherical albedo $A_{\mathrm{ct} 0}=A_{\mathrm{ct} 0}\left(\tau_{\mathrm{c} 0}, h_{\mathrm{t} 0}\right)$, where $\tau_{\mathrm{c} 0}$ is the optical thickness. Further, let $h_{\mathrm{t}}^{\star}$ solve the minimization problem

$$
h_{\mathrm{t}}^{\star}=\arg \min _{h_{\mathrm{t}}}\left[I_{\mathrm{ELC}}\left(A_{\mathrm{ct} 0}, h_{\mathrm{t}}\right)-I\left(A_{\mathrm{ct} 0}, h_{\mathrm{t} 0}\right)\right]^{2},
$$

where $I_{\mathrm{ELC}}\left(A_{\mathrm{ct} 0}, h_{\mathrm{t}}\right)$ is the radiance computed by the ELC model. The minimizer $h_{\mathrm{t}}^{\star}$ that yields a small residual, and so, an acceptable accuracy, is different from $h_{\mathrm{t} 0}$. Thus, the physical significance of the cloud top height is lost.
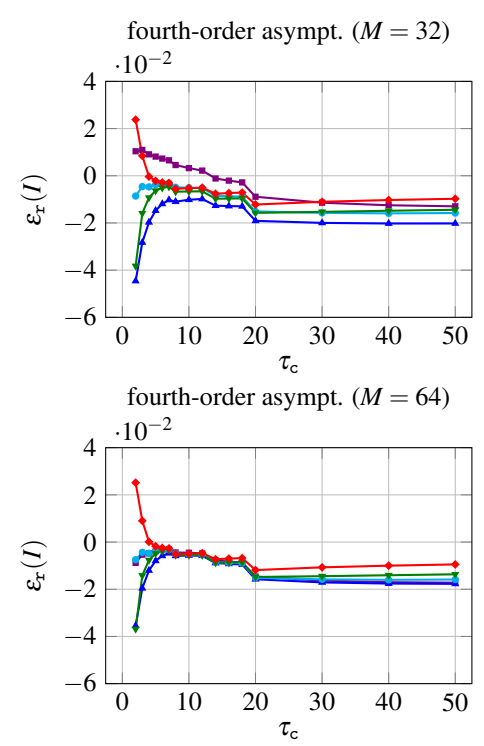
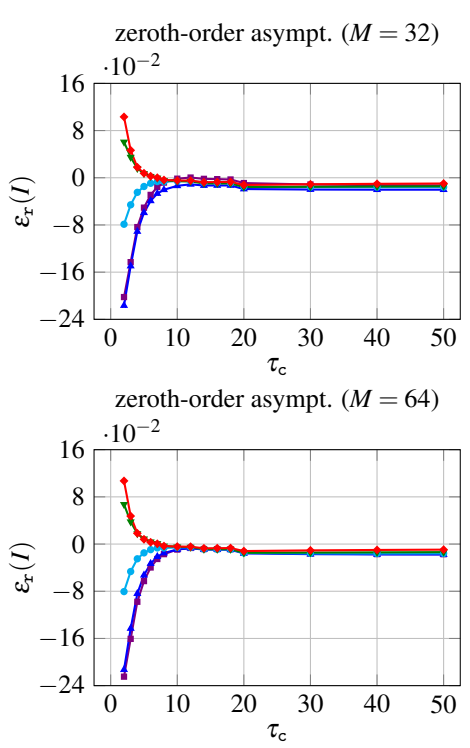

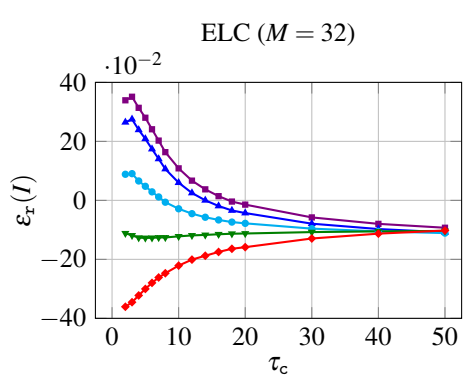

$\operatorname{ELC}(M=64)$

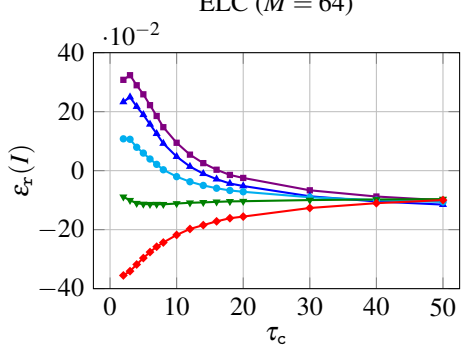

$\multimap \theta=5^{\circ} \multimap \theta=15^{\circ} \multimap \theta=30^{\circ} \multimap \theta=45^{\circ} \rightarrow \theta=60^{\circ}$

Figure 7: Relative errors in radiances as functions of $\tau_{\mathrm{c}}$ when using the approximate models. The results correspond to $M=32$ (top panels) and $M=64$ (bottom panels), and are computed by using the fourth-order asymptotic model, the zeroth-order asymptotic model and the ELC model. 

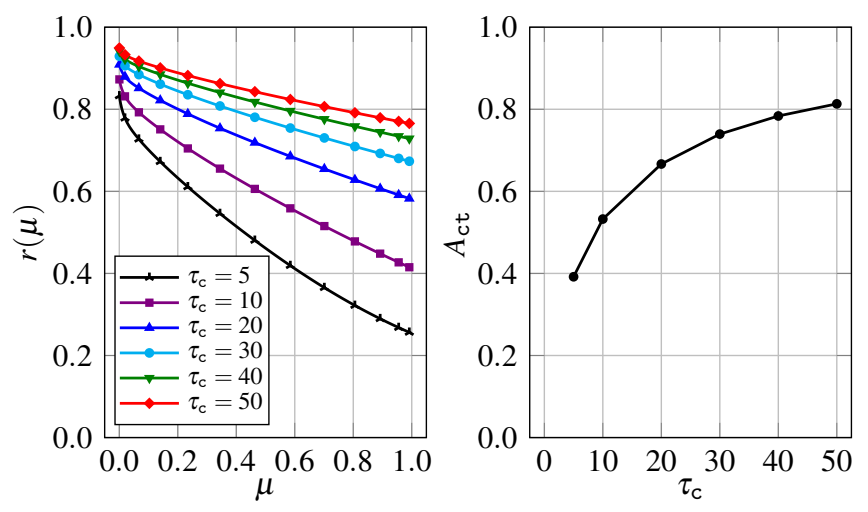

Figure 8: The plane albedo $r(\mu)$ for different values of $\tau_{\mathrm{c}}$ (left panel), and the spherical albedo $A_{\mathrm{ct}}$ as a function of $\tau_{\mathrm{c}}$ (right panel).
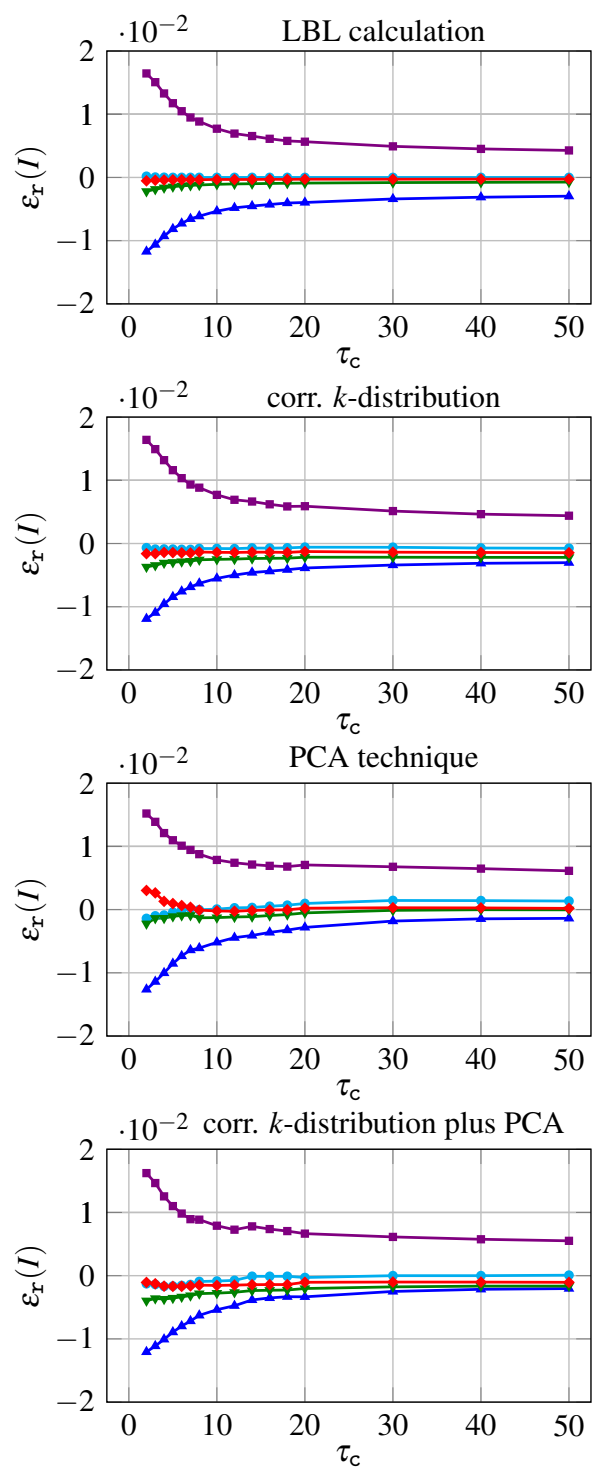

$\longrightarrow-\theta=5^{\circ} \multimap \theta=15^{\circ} \multimap \theta=30^{\circ} \multimap \theta=45^{\circ} \multimap \theta=60^{\circ}$

Figure 9: Relative errors in DOME radiances as functions of $\tau_{\mathrm{c}}$ when the acceleration techniques are used. The results correspond to $M=32$, and are computed by using LBL calculations, correlated $k$-distribution, PCA technique and correlated $k$-distribution plus PCA. Note that the first plot is the same one as the fourth plot of Fig. 5 .
Table 3: Computation user times in minutes for the results in Fig.9, and speedup factors with respect to the case of LBL calculation.

\begin{tabular}{lrr}
\hline Acceleration technique & $\mathbf{t} / \mathbf{m i n}$ & Speed-up factor \\
\hline LBL calculation & 1711 & - \\
Correlated $k$-distribution & 159 & 10.8 \\
PCA technique & 22 & 77.8 \\
Corr. $k$-distribution plus PCA technique & 5 & 342.2 \\
\hline
\end{tabular}

Table 4: Computation times in seconds for the approximate models using the combined correlated $k$-distribution plus PCA technique. The simulations correspond to the same scenario as in Fig. 9.

\begin{tabular}{lrr}
\hline Forward model & $\mathbf{t} / \mathbf{s}$ & Speed-up factor \\
\hline DOME & 285.0 & - \\
Fourth-order asymptotic & 221.8 & 1.3 \\
Zeroth-order asymptotic & 222.2 & 1.3 \\
ELC & 188.7 & 1.5 \\
\hline
\end{tabular}

\subsection{Acceleration techniques}

In Fig. 9 we illustrate the relative errors in DOME radiances when LBL calculations are replaced with the correlated $k$-distribution method, the PCA technique, and the combined correlated $k$-distribution plus PCA method. The corresponding computation times are given in Table 3 . Note that almost identical relative errors and computation times are obtained for MOME (not shown here). The general observation is that the relative error curves are practically unchanged when these acceleration techniques are used. The computation time using the combined correlated $k$-distribution plus PCA method is 5 times smaller than that for the PCA technique alone, and 40 times smaller than that for the correlated $k$-distribution method alone. Taking into account that the results in Table 3 correspond to 16 values of $\tau_{c}$, the average time to simultaneously compute the radiances at 5 viewing zenith angles is 17.8 seconds.

In Table 4 we show the computation times of the approximate models using the combined correlated $k$-distribution plus PCA method. As in Fig. 9, the radiances are computed for 16 values of $\tau_{\mathrm{c}}$ and at 5 viewing zenith angles. Note that the relative error curves are very similar to those plotted in the top panels of Fig. 7 (for $M=32$ ). The conclusion is that the computation times of the approximate models are lower, but not significantly lower than those of DOME.

\section{Conclusions}

We analyzed several radiative transfer models which can be used for the retrieval of cloud parameters from EPIC measurements. The radiative transfer models are the exact DOME and MOME models, and the approximate asymptotic and equivalent Lambertian cloud models. MOME is a combination of the conventional matrix operator method and DOME. Essentially, the matrix exponential formalism enabled us to combine the two methods in a simple way and to introduce the higher-order 
asymptotic and equivalent Lambertian cloud models in a natural manner. The radiative transfer models used several acceleration techniques such as the Padé approximation, the telescoping technique, the method of false discrete ordinate, the correlated $k$-distribution method and the PCA technique. We computed the radiances also by combining the correlated $k$-distribution method with the PCA technique.

We analyzed the accuracy and efficiency of the radiative transfer models when simulating radiances for the channel 9 of the EPIC instrument. In summary, the following conclusions can be drawn:

1. The exact DOME and MOME models using correlated $k$-distribution in conjunction with PCA yield an accuracy better than 0.015 and a computation time of approximately 18 seconds for radiance calculations at 5 viewing zenith angles.

2. The approximate models are slightly more efficient than the exact models but their accuracy leaves much to be desired. In particular, the relative errors of the zeroth-order asymptotic model are large for cloud optical thicknesses smaller than 10, while the relative errors of the ELC model are large for all cloud optical thicknesses.

Thus, it appears that the exact DOME and MOME models could fulfill the accuracy and efficiency requirements of an offline processor for retrieval of cloud parameters from EPIC measurements. For online (i.e. operational) retrieval algorithms, the inversion is frequently performed by using look-up table (LUT) approaches $[4,5,42,43]$. The size of such a LUT is extremely large, and so the time for computing a LUT by using exact radiative transfer models is normally too high. Even though the inversion is performed by neural network techniques [7, 8, 44], the time for neural network training by using exact radiative transfer models is high. In this regard, the above radiative transfer models are an efficient tool for LUTs computation and neural network training.

\section{Acknowledgements}

This work was funded by the programme DLR/DAAD Research Fellowships 2015 (57186656), with reference numbers 91613528 and 91627488 , organized by the German Academic Exchange Service (DAAD) and the German Aerospace Center (DLR).

\section{Appendix A}

In this appendix we give the expressions for the layer quantities $\mathrm{A}_{m j}^{1}, \mathrm{~A}_{m j}^{2}$ and $\mathrm{b}_{m j}$ in (13) computed by the eigendecomposition method and the Padé approximation.

The eigendecomposition method for computing the matrix exponential is based on a spectral decomposition of the matrix $\mathbf{A}_{m j}$ given by (8). This can be obtained by one of the following methods: direct decomposition of an asymmetric matrix [45], square-root decomposition [46], and Cholesky decomposition
[47]. Here, we use the direct decomposition method. Exploiting the block symmetry of $\mathbf{A}_{m j}$, we find

$$
\mathbf{A}_{m j}=\mathbf{V}_{m j}\left[\begin{array}{cc}
\boldsymbol{\Lambda}_{m j} & \mathbf{0} \\
\mathbf{0} & -\boldsymbol{\Lambda}_{m j}
\end{array}\right] \mathbf{V}_{m j}^{-1}
$$

with

$$
\begin{aligned}
& \mathbf{V}_{m j}=\left[\begin{array}{cc}
\mathbf{V}_{m j}^{+} & \mathbf{V}_{m j}^{-} \\
\mathbf{V}_{m j}^{-} & \mathbf{V}_{m j}^{+}
\end{array}\right], \\
& \mathbf{V}_{m j}^{ \pm}=\left[\mathbf{v}_{1}^{ \pm}, \ldots, \mathbf{v}_{M}^{ \pm}\right],
\end{aligned}
$$

and

$$
\Lambda_{m j}=\operatorname{diag}\left[\lambda_{1}, \ldots, \lambda_{M}\right] \stackrel{\text { not }}{=} \operatorname{diag}\left[\lambda_{k}\right]
$$

Note that $\left[\begin{array}{c}\mathbf{v}_{k}^{+} \\ \mathbf{v}_{k}^{-}\end{array}\right]$are the right eigenvectors of $\mathbf{A}_{m j}$ corresponding to the eigenvalue $\lambda_{k}$, and $\left[\begin{array}{c}\mathbf{v}_{k}^{-} \\ \mathbf{v}_{k}^{+}\end{array}\right]$are the right eigenvectors of $\mathbf{A}_{m j}$ corresponding to the eigenvalue $-\lambda_{k}$. As a result, the layer matrices can be expressed in compact form as

$$
\begin{aligned}
& \mathbf{A}_{m j}^{1}=\mathbf{D}_{m j}^{1} \mathbf{V}_{m j}^{-1}, \\
& \mathbf{A}_{m j}^{2}=-\mathbf{D}_{m j}^{2} \mathbf{V}_{m j}^{-1},
\end{aligned}
$$

where the diagonal scaling matrices $\mathbf{D}_{m j}^{1}$ and $\mathbf{D}_{m j}^{2}$ are given by

$$
\begin{aligned}
& \mathbf{D}_{m j}^{1}=\operatorname{diag}\left[a_{0}\left(\lambda_{k} \Delta r_{j}\right) ; 1\right], \\
& \mathbf{D}_{m j}^{2}=\operatorname{diag}\left[1 ; a_{0}\left(\lambda_{k} \Delta r_{j}\right)\right],
\end{aligned}
$$

with $a_{0}(x)=\mathrm{e}^{-x}$. The layer vector is computed as

$$
\mathbf{b}_{m j}=\mathbf{B}_{m j} \mathbf{V}_{m j}^{-1}\left(\Delta r_{j} \mathbf{b}_{m j}\right),
$$

where, in the secant approximation of the solar beam, the diagonal scaling matrix $\mathbf{B}_{m j}$ is given by

$$
\mathbf{B}_{m j}=\operatorname{diag}\left[b_{1}\left(\lambda_{k} \Delta r_{j}\right) ; b_{2}\left(\lambda_{k} \Delta r_{j}\right)\right],
$$

with

$$
\begin{aligned}
& b_{1}(x)=\frac{\mathrm{e}^{-\left(\tau_{\text {ext } j}^{0}+x\right)}-\mathrm{e}^{-\tau_{\text {ext } j+1}^{0}}}{\tau_{\text {ext } j+1}^{0}-\tau_{\text {ext } j}^{0}-x}, \\
& b_{2}(x)=\frac{\mathrm{e}^{-\left(\tau_{\text {ext } j+1}^{0}+x\right)}-\mathrm{e}^{-\tau_{\text {ext } j}^{0}}}{\tau_{\text {ext } j}^{0}-\tau_{\text {ext } j+1}^{0}-x}
\end{aligned}
$$

In (48) and (49), $\tau_{\text {ext } j}^{0}$ and $\tau_{\text {ext } j+1}^{0}$ are the solar optical depths at the boundary levels $j$ and $j+1$, respectively.

In the first-order Padé approximation to the matrix exponential, the layer equation reads as

$$
\begin{aligned}
& \mathbf{A}_{m j}^{1}=\mathbf{I}-\frac{\Delta r_{j}}{2} \mathbf{A}_{m j}, \\
& \mathrm{~A}_{m j}^{2}=-\left(\mathbf{I}+\frac{\Delta r_{j}}{2} \mathbf{A}_{m j}\right), \\
& \mathbf{b}_{m j}=\mathbf{B}_{m j}\left(\Delta r_{j} \mathbf{b}_{m j}\right) \mathrm{e}^{-\tau_{\text {ext } j}^{0},}
\end{aligned}
$$


where $\mathbf{B}_{m j}$ is now given by

$$
\mathbf{B}_{m j}=I_{0}\left(\tau_{0}\right) \mathbf{I}+\left[\frac{1}{2} I_{0}\left(\tau_{0}\right)-I_{1}\left(\tau_{0}\right)\right] \Delta r_{j} \mathbf{A}_{m j},
$$

with $\tau_{0}=\tau_{\text {ext } j+1}^{0}-\tau_{\text {ext } j}^{0}$ and

$$
\begin{aligned}
& I_{0}(x)=\frac{1-\mathrm{e}^{-x}}{x}, \\
& I_{1}(x)=\frac{1}{x}\left[1-\frac{\mathrm{e}^{-x}}{x}\left(1-\mathrm{e}^{-x}\right)\right] .
\end{aligned}
$$

\section{Appendix B}

In this appendix we give the expressions of $\mathbf{R}_{m j}, \mathbf{T}_{m j}$ and $\boldsymbol{\Sigma}_{m j}$ in (19) computed by the eigendecomposition method and the Padé approximation.

The reflection and transmission matrices of layer $j$ can be derived by representing the layer equation (13) as

$$
\left[\begin{array}{c}
\mathbf{i}_{m j}^{+} \\
\mathbf{i}_{m j+1}^{-}
\end{array}\right]=\left[\begin{array}{cc}
\mathbf{R}_{m j} & \mathbf{T}_{m j} \\
\mathbf{T}_{m j} & \mathbf{R}_{m j}
\end{array}\right]\left[\begin{array}{c}
\mathbf{i}_{m j}^{-} \\
\mathbf{i}_{m j+1}^{+}
\end{array}\right]+\left[\begin{array}{c}
\boldsymbol{\Sigma}_{m j}^{+} \\
\boldsymbol{\Sigma}_{m j}^{-}
\end{array}\right],
$$

from which we find that

$$
\begin{aligned}
{\left[\begin{array}{cc}
\mathbf{R}_{m j} & \mathbf{T}_{m j} \\
\mathbf{T}_{m j} & \mathbf{R}_{m j}
\end{array}\right]=} & -\left[\begin{array}{cc}
\Gamma_{m j} \mathbf{V}_{m j}^{11} & -\mathbf{V}_{m j}^{12} \\
\mathbf{V}_{m j}^{21} & -\Gamma_{m j} \mathbf{V}_{m j}^{22}
\end{array}\right]^{-1} \\
& \times\left[\begin{array}{cc}
\Gamma_{m j} \mathbf{V}_{m j}^{12} & -\mathbf{V}_{m j}^{11} \\
\mathbf{V}_{m j}^{22} & -\Gamma_{m j} \mathbf{V}_{m j}^{21}
\end{array}\right], \\
{\left[\begin{array}{c}
\boldsymbol{\Sigma}_{m j}^{+} \\
\boldsymbol{\Sigma}_{m j}^{-}
\end{array}\right]=} & {\left[\begin{array}{cc}
\Gamma_{m j} \mathbf{V}_{m j}^{11} & -\mathbf{V}_{m j}^{12} \\
\mathbf{V}_{m j}^{21} & -\Gamma_{m j} \mathbf{V}_{m j}^{22}
\end{array}\right]^{-1} \mathrm{~b}_{m j}, }
\end{aligned}
$$

with

$$
\mathbf{V}_{m j}^{-1}=\left[\begin{array}{cc}
\mathbf{V}_{m j}^{11} & \mathbf{V}_{m j}^{12} \\
\mathbf{V}_{m j}^{22} & \mathbf{V}_{m j}^{22}
\end{array}\right]
$$

and

$$
\Gamma_{m j}=\operatorname{diag}\left[\exp \left(-\lambda_{k} \Delta r_{j}\right)\right]
$$

By inspection of (57) and (58) it is apparent that the computation of $\mathbf{R}_{m j}, \mathbf{T}_{m j}$ and $\boldsymbol{\Sigma}_{m j}$ requires an inversion and a multiplication of matrices of dimension $2 M \times 2 M$. Other methods in which the computation of $\mathbf{R}_{m j}$ and $\mathbf{T}_{m j}$ can be halved in order are discussed in [32].

In the first-order Padé approximation to the matrix exponential, we have

$$
\begin{aligned}
{\left[\begin{array}{cc}
\mathbf{R}_{m j} & \mathbf{T}_{m j} \\
\mathbf{T}_{m j} & \mathbf{R}_{m j}
\end{array}\right]=} & -\left[\begin{array}{cc}
\mathbf{I}-\frac{1}{2} \Delta r_{j} \mathbf{A}_{m j}^{11} & -\frac{1}{2} \Delta r_{j} \mathbf{A}_{m j}^{12} \\
\frac{1}{2} \Delta r_{j} \mathbf{A}_{m j}^{12} & -\left(\mathbf{I}-\frac{1}{2} \Delta r_{j} \mathbf{A}_{m j}^{11}\right)
\end{array}\right]^{-1} \\
& \times\left[\begin{array}{cc}
-\frac{1}{2} \Delta r_{j} \mathbf{A}_{m j}^{12} & -\left(\mathbf{I}+\frac{1}{2} \Delta r_{j} \mathbf{A}_{m j}^{11}\right) \\
\mathbf{I}+\frac{1}{2} \Delta r_{j} \mathbf{A}_{m j}^{11} & \frac{1}{2} \Delta r_{j} \mathbf{A}_{m j}^{12}
\end{array}\right], \\
{\left[\begin{array}{c}
\boldsymbol{\Sigma}_{m j}^{+} \\
\boldsymbol{\Sigma}_{m j}^{-}
\end{array}\right]=} & {\left[\begin{array}{cc}
\mathbf{I}-\frac{1}{2} \Delta r_{j} \mathbf{A}_{m j}^{11} & -\frac{1}{2} \Delta r_{j} \mathbf{A}_{m j}^{12} \\
\frac{1}{2} \Delta r_{j} \mathbf{A}_{m j}^{12} & -\left(\mathbf{I}-\frac{1}{2} \Delta r_{j} \mathbf{A}_{m j}^{11}\right)
\end{array}\right]^{-1} \mathrm{~b}_{m j} . }
\end{aligned}
$$

\section{Appendix C}

In this appendix we give a justification of the ELC model.

For the azimuthal mode $m$, the reflection law at the cloud top height is (we omit the $r_{j_{\min }}$ dependency)

$$
I_{m}(\mu)=2 \int_{0}^{1} R_{m}\left(\mu, \mu^{\prime}\right) I_{\mathrm{t} m}\left(-\mu^{\prime}\right) \mu^{\prime} \mathrm{d} \mu^{\prime}, \quad m \geq 0,
$$

where $I_{m}(\mu)$ is the upward diffuse radiance in the direction $\mu$, $R_{m}\left(\mu, \mu^{\prime}\right)$ is the reflection function of the atmosphere below the cloud, and $I_{\mathrm{t} m}\left(-\mu^{\prime}\right)$ is the downward total radiance. The upward flux is

$$
E^{\uparrow}=\int_{0}^{2 \pi} \int_{0}^{1} I(\mu, \varphi) \mu \mathrm{d} \mu \mathrm{d} \varphi=2 \pi \int_{0}^{1} r\left(\mu^{\prime}\right) I_{\mathrm{t} 0}\left(-\mu^{\prime}\right) \mu^{\prime} \mathrm{d} \mu^{\prime},
$$

where

$$
r(\mu)=2 \int_{0}^{1} R_{0}\left(\mu, \mu^{\prime}\right) \mu^{\prime} \mathrm{d} \mu^{\prime}=2 \int_{0}^{1} R_{0}\left(\mu^{\prime}, \mu\right) \mu^{\prime} \mathrm{d} \mu^{\prime}
$$

is the plane albedo of the atmosphere below the cloud. Let us assume that the plane albedo $r(\mu)$ is almost constant with respect to $\mu$, so that we can write $r(\mu)=r_{0}$. Then, the spherical albedo of the atmosphere below the cloud is

$$
A_{\mathrm{ct}}=2 \int_{0}^{1} r(\mu) \mu \mathrm{d} \mu=r_{0},
$$

and the upward flux is

$$
E^{\uparrow}=2 \pi A_{\mathrm{ct}} \int_{0}^{1} I_{\mathrm{t} 0}\left(-\mu^{\prime}\right) \mu^{\prime} \mathrm{d} \mu^{\prime} .
$$

The upward flux given by (67) is that of a Lambertian surface with a ground albedo equal to the spherical albedo $A_{\mathrm{ct}}$. By replacing the atmosphere below the cloud by a Lambertian surface with the reflection function $R_{m}\left(\mu, \mu^{\prime}\right)=A_{\mathrm{ct}} \delta_{m 0}$, we try to ensure the conservation of radiative flux. However, this is possible only when the plane albedo $r(\mu)$ does not vary significantly with $\mu$.

\section{Appendix D}

In this appendix we describe the telescoping technique for DOME and MOME.

Let us make the assumption that for all layers $j$, with $j<j_{\text {min }}$ and $j>j_{\max }$, and all azimuthal modes $m>2$, the azimuthal expansion coefficients of the phase functions vanish. In this case, $\mathbf{A}_{m j}$ is a diagonal matrix,

$$
\mathbf{A}_{m j}=\operatorname{diag}\left[-\frac{\sigma_{\text {ext } j}}{\mu_{k}} ; \frac{\sigma_{\text {ext } j}}{\mu_{k}}\right],
$$

and the solar layer vector $\mathbf{b}_{m j}$ vanishes. The layer equation simplifies to

$$
\mathbf{i}_{m j+1}=\mathrm{e}^{-\mathbf{A}_{m j} \Delta r_{j}} \mathbf{i}_{m j}
$$


which further implies that

$$
\begin{aligned}
\mathbf{i}_{m j}^{+} & =\operatorname{diag}\left[\exp \left(-\frac{\sigma_{\text {ext } j} \Delta r_{j}}{\mu_{k}}\right)\right] \mathbf{i}_{m j+1}^{+}, \\
\mathbf{i}_{m j+1}^{-} & =\operatorname{diag}\left[\exp \left(-\frac{\sigma_{\text {ext } j \Delta r_{j}}}{\mu_{k}}\right)\right] \mathbf{i}_{m j}^{-} .
\end{aligned}
$$

In the matrix operator method, we have

$$
\mathbf{R}_{m j}=0, \quad \mathbf{T}_{m j}=\operatorname{diag}\left[\exp \left(-\frac{\sigma_{\text {ext } j} \triangle r_{j}}{\mu_{k}}\right)\right], \quad \boldsymbol{\Sigma}_{m j}^{ \pm}=0,
$$

and the adding formula becomes

$$
\begin{aligned}
& \mathbf{R}_{m j}=\mathbf{T}_{m j} \mathbf{R}_{m j+1} \mathbf{T}_{m j}, \\
& \mathbf{r}_{m j}=\mathbf{T}_{m j} \mathbf{r}_{m j+1} .
\end{aligned}
$$

Let

$$
\mathrm{T}=\operatorname{diag}\left[\exp \left(-\frac{1}{\mu_{k}} \sum_{j=j_{\max }+1}^{N-1} \sigma_{\text {ext } j} \Delta r_{j}\right)\right]
$$

be the transmission matrix of the layers below the cloud. The DOME telescoping technique can be summarized as follows. For $m>2$,

1. solve the radiative transfer equation for the cloud layers with the top and bottom boundary conditions

$$
\mathbf{i}_{m, j_{\min }}^{-}=0
$$

and

$$
\begin{aligned}
\mathbf{i}_{m j_{\max }+1}^{+} & =\mathrm{R}_{m j_{\max }+1} \mathbf{i}_{m j_{\max }+1}^{-}+\mathrm{r}_{m j_{\max }+1}, \\
\mathrm{R}_{m j_{\max }+1} & =\mathrm{T} \mathrm{R}_{m N} \mathrm{~T}, \\
\mathrm{r}_{m j_{\max }+1} & =\mathrm{T}_{m N},
\end{aligned}
$$

respectively;

2. set $\mathbf{i}_{m j}^{-}=0$ for $j_{\min }-1 \leq j \leq 1$, and compute the radiances above the cloud and in the upward directions $\mathbf{i}_{m j}^{+}$for $j_{\text {min }}-1 \leq j \leq 1$, by using recurrence $(70)$;

3 . compute the radiances below the cloud and in the downward directions $\mathbf{i}_{m j+1}^{-}$for $j_{\max }+1 \leq j \leq N-1$, by using recurrence (71);

4. compute the upward radiance at the surface $\mathbf{i}_{m N}^{+}$from the boundary condition (15);

5. compute the radiances below the cloud and in the upward directions $\mathbf{i}_{m j}^{+}$for $j_{\max }+2 \leq j \leq N-1$, by using recurrence (70).

The MOME telescoping technique involves the following steps. For $m>2$,

1. compute the reflection matrix $\mathrm{R}_{m j_{\max }+1}$ and the reflection vector $\mathbf{r}_{m j_{\max }+1}$ at the cloud bottom by means of (77) and (78), respectively;

2. compute $\mathrm{R}_{m j_{\min }}$ and $\mathrm{r}_{m j_{\min }}$ by using the adding algorithm;

3. set $\mathbf{i}_{m j}^{-}=0$ for $j_{\min } \leq j \leq 1$, and $\mathbf{i}_{m j_{\min }}^{+}=\mathbf{r}_{m j_{\min }}$ (boundary condition at the cloud top);

4. compute the radiances above the cloud and in the upward directions $\mathbf{i}_{m j}^{+}$for $j_{\min }-1 \leq j \leq 1$, by using recurrence (70).

\section{References}

[1] A. Marshak, Y. Knyazikhin, The spectral invariant approximation within canopy radiative transfer to support the use of the EPIC/DSCOVR oxygen B-band for monitoring vegetation, Journal of Quantitative Spectroscopy and Radiative Transfer 191 (2017) 7-12.

doi:10.1016/j.jqsrt.2017.01.015

[2] X. Xu, J. Wang, Y. Wang, J. Zeng, O. Torres, Y. Yang, A. Marshak, J. Reid, S. Miller, Passive remote sensing of altitude and optical depth of dust plumes using the oxygen A and B bands: First results from EPIC/DSCOVR at Lagrange-1 point, Geophysical Research Letters 44 (14) (2017) 7544-7554. doi:10.1002/2017gl073939

[3] Y. Yang, A. Marshak, J. Mao, A. Lyapustin, J. Herman, A method of retrieving cloud top height and cloud geometrical thickness with oxygen A and B bands for the Deep Space Climate Observatory (DSCOVR) mission: Radiative transfer simulations, Journal of Quantitative Spectroscopy and Radiative Transfer 122 (2013) 141-149. doi:10.1016/j.jqsrt. 2012.09.017

[4] S. Platnick, M. King, S. Ackerman, W. Menzel, B. Baum, J. Rièdi, R. Frey, The MODIS cloud products: Algorithms and examples from Terra, IEEE Transactions on Geoscience and Remote Sensing 41 (2003) 459-473. doi:10.1109/tgrs.2002.808301

[5] M. King, S.-C. Tsay, S. Platnick, M. Wang, K.-N. Liou, Cloud retrieval algorithms for MODIS: Optical thickness, effective particle radius, and thermodynamic phase, Tech. rep., National Aeronautics and Space Administration, United States, available at https://eospso.nasa.gov/ sites/default/files/atbd/atbd_mod05.pdf (1997).

[6] A. Kokhanovsky, V. Rozanov, P. Zege, H. Bovensmann, J. Burrows, A semianalytical cloud retrieval algorithm using backscattered radiation in 0.4-2.4 $\mu$ m spectral region, Journal of Geophysical Research 108 (2003) AAC 4-1-AAC 4-19. doi: $10.1029 / 2001$ jd001543

[7] D. Loyola, W. Thomas, Y. Livschitz, T. Ruppert, P. Albert, R. Hollmann, Cloud properties derived from GOME/ERS-2 backscatter data for trace gas retrieval, IEEE Transactions on Geoscience and Remote Sensing 45 (9) (2007) 2747-2758.

doi:10.1109/tgrs.2007.901043

[8] D. Loyola R., W. Thomas, R. Spurr, B. Mayer, Global patterns in daytime cloud properties as derived from GOME backscatter UV-VIS measurements, International Journal of Remote Sensing 31 (16) (2010) 42954318. doi: 10.1080/01431160903246741

[9] R. B. A. Koelemeijer, P. Stammes, J. W. Hovenier, J. F. de Haan, A fast method for retrieval of cloud parameters using oxygen A band measurements from the Global Ozone Monitoring Experiment, Journal of Geophysical Research 106 (D4) (2001) 3475. doi: $10.1029 / 2000 j d 900657$

[10] R. Goody, R. West, L. Chen, D. Crisp, The correlated k-method for radiation calculations in nonhomogeneous atmospheres, Journal of Quantitative Spectroscopy and Radiative Transfer 42 (6) (1989) 539-550. doi: 10.1016/0022-4073(89) 90044-7

[11] W. Wiscombe, J. Evans, Exponential-sum fitting of radiative transmission functions, Journal of Computational Physics 24 (4) (1977) 416-444. doi : 10.1016/0021-9991(77) 90031-6

[12] S. A. Tjemkes, J. Schmetz, Synthetic satellite radiances using the radiance sampling method, Journal of Geophysical Research: Atmospheres 102 (D2) (1997) 1807-1818. doi: 10.1029/96jd02684

[13] R. West, D. Crisp, L. Chen, Mapping transformations for broadband atmospheric radiation calculations, Journal of Quantitative Spectroscopy and Radiative Transfer 43 (3) (1990) 191-199. doi:10.1016/0022-4073(90)90051-7

[14] X. Liu, W. L. Smith, D. K. Zhou, A. Larar, Principal component-based radiative transfer model for hyperspectral sensors: Theoretical concept, Applied Optics 45 (1) (2006) 201. doi:10.1364/ao.45.000201

[15] P. Eriksson, C. Jiménez, S. Bühler, D. Murtagh, A Hotelling transformation approach for rapid inversion of atmospheric spectra, Journal of Quantitative Spectroscopy and Radiative Transfer 73 (6) (2002) 529-543. doi: 10.1016/s0022-4073(01)00175-3 
[16] V. Natraj, X. Jiang, R. Shia, X. Huang, J. Margolis, Y. Yung, Application of the principal component analysis to high spectral resolution radiative transfer: A case study of the $\mathrm{O}_{2}$ A-band, Journal of Quantitative Spectroscopy and Radiative Transfer 95 (4) (2005) 539-556. doi:10.1016/j.jqsrt.2004.12.024

[17] V. Natraj, R. Shia, Y. Yung, On the use of principal component analysis to speed up radiative transfer calculations, Journal of Quantitative Spectroscopy and Radiative Transfer 111 (5) (2010) 810-816. doi:10.1016/j.jqsrt.2009.11.004

[18] D. Efremenko, A. Doicu, D. Loyola, T. Trautmann, Optical property dimensionality reduction techniques for accelerated radiative transfer performance: Application to remote sensing total ozone retrievals, Journal of Quantitative Spectroscopy and Radiative Transfer 133 (2014) 128-135. doi:10.1016/j.jqsrt.2013.07.023

[19] R. Spurr, LIDORT and VLIDORT. Linearized pseudo-spherical scalar and vector discrete ordinate radiative transfer models for use in remote sensing retrieval problems, in: A. Kokhanovsky (Ed.), Light scattering reviews, Vol. 3, 2008, pp. 229-275. doi: $10.1007 / 978-3-540-48546-9$-7

[20] A. Doicu, T. Trautmann, Discrete-ordinate method with matrix exponential for a pseudo-spherical atmosphere: Scalar case, Journal of Quantitative Spectroscopy and Radiative Transfer 110 (1-2) (2009) 146-158. doi:10.1016/j.jqsrt.2008.09.014

[21] Q. Liu, F. Weng, Advanced doubling-adding method for radiative transfer in planetary atmospheres, Journal of the Atmospheric Sciences 63 (12) (2006) 3459-3465. doi:10.1175/jas3808.1

[22] K. Evans, G. Stephens, A new polarized atmospheric radiative transfer model, Journal of Quantitative Spectroscopy and Radiative Transfer 46 (5) (1991) 413-423. doi : 10.1016/0022-4073(91)90043-p

[23] E. Chalhoub, R. Garcia, The equivalence between two techniques of angular interpolation for the discrete-ordinates method, Journal of Quantitative Spectroscopy and Radiative Transfer 64 (5) (2000) 517-535. doi:10.1016/s0022-4073(99)00134-x

[24] D. Efremenko, A. Doicu, D. Loyola, T. Trautmann, Acceleration techniques for the discrete ordinate method, Journal of Quantitative Spectroscopy and Radiative Transfer 114 (2013) 73-81. doi:10.1016/j.jqsrt.2012.08.014

[25] B. Mayer, M. Schröder, R. Preusker, L. Schüller, Remote sensing of water cloud droplet size distributions using the backscatter glory: A case study, Atmospheric Chemistry and Physics 4 (5) (2004) 1255-1263. doi : $10.5194 / \mathrm{acp}-4-1255-2004$

[26] W. Wiscombe, The delta-M method: Rapid yet accurate radiative flux calculations for strongly asymmetric phase functions, Journal of the Atmospheric Sciences 34 (9) (1977) 1408-1422. doi : 10.1175/1520-0469(1977)034<1408: tdmrya>2.0.co;2

[27] R. Spurr, Simultaneous derivation of intensities and weighting functions in a general pseudo-spherical discrete ordinate radiative transfer treatment, Journal of Quantitative Spectroscopy and Radiative Transfer 75 (2) (2002) 129-175. doi:10.1016/s0022-4073(01)00245-x

[28] A. Doicu, T. Trautmann, Discrete-ordinate method with matrix exponential for a pseudo-spherical atmosphere: Vector case, Journal of Quantitative Spectroscopy and Radiative Transfer 110 (1-2) (2009) 159-172. doi:10.1016/j.jqsrt.2008.09.013

[29] R. Spurr, M. Christi, Linearization of the interaction principle: Analytic jacobians in the "radiant model", Journal of Quantitative Spectroscopy and Radiative Transfer 103 (3) (2007) 431-446. doi:10.1016/j.jqsrt.2006.05.001

[30] E. Yanovitskij, Light scattering in inhomogeneous atmospheres, SpringerVerlag, 1997.

[31] T. Nakajima, M. D. King, Asymptotic theory for optically thick layers: Application to the discrete ordinates method, Applied Optics 31 (36) (1992) 7669-7683. doi: 10.1364/ao.31.007669

[32] D. S. Efremenko, V. Molina García, S. Gimeno García, A. Doicu, A review of the matrix-exponential formalism in radiative transfer, Journal of Quantitative Spectroscopy and Radiative Transfer 196 (2017) 17-45. doi:10.1016/j.jqsrt.2017.02.015

[33] M. D. King, Determination of the scaled optical thickness of clouds from reflected solar radiation measurements, Journal of the Atmospheric Sciences 44 (13) (1987) 1734-1751.

doi : 10.1175/1520-0469(1987)044<1734: dotsot>2.0.co;2

[34] T. Nakajima, M. Tanaka, Algorithms for radiative intensity calculations in moderately thick atmospheres using a truncation approximation, Journal of Quantitative Spectroscopy and Radiative Transfer 40 (1) (1988) 5169. doi: $10.1016 / 0022-4073(88) 90031-3$

[35] V. Ambartsumian, The effect of the absorption lines on the radiative equilibrium of the outer layers of the stars, Publications of the Astronomical Observatory of Leningrad State University 6 (1936) 7-18.

[36] K. Stamnes, S.-C. Tsay, I. Laszlo, DISORT, a general-purpose Fortran program for discrete-ordinate-method radiative transfer in scattering and emitting layered media: Documentation of methodology, Tech. rep., Dept. of Physics and Engineering Physics, Stevens Institute of Technology, Hoboken, NJ 07030, available at http: //www. libradtran.org/ lib/exe/fetch.php?media=disortreport1.1.pdf (2000).

[37] F. Schreier, S. Gimeno García, P. Hedelt, M. Hess, J. Mendrok, M. Vasquez, J. Xu, GARLIC - A general purpose atmospheric radiative transfer line-by-line infrared-microwave code: Implementation and evaluation, Journal of Quantitative Spectroscopy and Radiative Transfer 137 (2014) 29-50. doi: $10.1016 / j \cdot j$.jsrt.2013.11.018

[38] F. Schreier, Optimized implementations of rational approximations for the Voigt and complex error function, Journal of Quantitative Spectroscopy and Radiative Transfer 112 (6) (2011) 1010-1025.

doi:10.1016/j.jqsrt.2010.12.010

[39] L. Rothman, I. Gordon, A. Barbe, D. C. Benner, P. Bernath, M. Birk, V. Boudon, L. Brown, A. Campargue, J.-P. Champion, K. Chance, L. Coudert, V. Dana, V. Devi, S. Fally, J.-M. Flaud, R. Gamache, A. Goldman, D. Jacquemart, I. Kleiner, N. Lacome, W. Lafferty, J.-Y. Mandin, S. Massie, S. Mikhailenko, C. Miller, N. Moazzen-Ahmadi, O. Naumenko, A. Nikitin, J. Orphal, V. Perevalov, A. Perrin, A. Predoi-Cross, C. Rinsland, M. Rotger, M. Šimečková, M. Smith, K. Sung, S. Tashkun, J. Tennyson, R. Toth, A. Vandaele, J. Vander Auwera, The HITRAN 2008 molecular spectroscopic database, Journal of Quantitative Spectroscopy and Radiative Transfer 110 (9-10) (2009) 533-572. doi:10.1016/j.jqsrt.2009.02.013

[40] B. A. Bodhaine, N. B. Wood, E. G. Dutton, J. R. Slusser, On Rayleigh optical depth calculations, Journal of Atmospheric and Oceanic Technology 16 (11) (1999) 1854-1861. doi: 10.1175/1520-0426(1999)016<1854: orodc>2.0.co;2

[41] G. Anderson, S. Clough, F. Kneizys, J. Chetwynd, E. Shettle, AFGL Atmospheric Constituent Profiles (0-120 km), Air Force Geophysics Laboratory, Hanscom Air Force Base, MA, USA, 1986.

[42] T. Y. Nakajima, T. Nakajma, Wide-area determination of cloud microphysical properties from NOAA AVHRR measurements for FIRE and ASTEX regions, Journal of the Atmospheric Sciences 52 (23) (1995) 4043-4059.

doi: 10.1175/1520-0469(1995) 052<4043: wadocm>2.0.co;2

[43] K. Kawamoto, T. Nakajima, T. Y. Nakajima, A global determination of cloud microphysics with AVHRR remote sensing, Journal of Climate 14 (9) (2001) 2054-2068. doi: 10.1175/1520-0442(2001) 014<2054: agdocm>2.0.co;2

[44] D. S. Efremenko, D. G. Loyola R., P. Hedelt, R. J. D. Spurr, Volcanic $\mathrm{SO}_{2}$ plume height retrieval from UV sensors using a full-physics inverse learning machine algorithm, International Journal of Remote Sensing 38 (sup1) (2017) 1-27. doi: $10.1080 / 01431161.2017 .1348644$

[45] K. Stamnes, R. A. Swanson, A new look at the discrete ordinate method for radiative transfer calculations in anisotropically scattering atmospheres, Journal of the Atmospheric Sciences 38 (2) (1981) 387-399. doi: 10.1175/1520-0469(1981)038<0387: anlatd>2.0.co;2

[46] T. Nakajima, M. Tanaka, Matrix formulations for the transfer of solar radiation in a plane-parallel scattering atmosphere, Journal of Quantitative Spectroscopy and Radiative Transfer 35 (1) (1986) 13-21. doi : 10.1016/0022-4073(86)90088-9

[47] K. Stamnes, S.-C. Tsay, W. Wiscombe, K. Jayaweera, Numerically stable algorithm for discrete-ordinate-method radiative transfer in multiple scattering and emitting layered media, Applied Optics 27 (12) (1988) 2502. doi:10.1364/ao.27.002502 